\title{
OT1OT1
}

\section{EFFECTIVE CRITERIA FOR SPECIFIC IDENTIFIABILITY OF TENSORS AND FORMS*}

\author{
LUCA CHIANTINI ${ }^{\dagger}$, GIORGIO OTTAVIANI ${ }^{\ddagger}$, AND NICK VANNIEUWENHOVEN ${ }^{\S}$
}

\begin{abstract}
In applications where the tensor rank decomposition arises, one often relies on its identifiability properties for interpreting the individual rank-1 terms appearing in the decomposition. Several criteria for identifiability have been proposed in the literature, but few results exist on how frequently they are satisfied. We propose to call a criterion effective if it is satisfied on a dense, open subset of the smallest semi-algebraic set enclosing the set of rank- $r$ tensors. We analyze the effectiveness of Kruskal's criterion when it is combined with reshaping. It is proved that this criterion is effective for both real and complex tensors in its entire range of applicability, which is usually much smaller than the smallest typical rank. Our proof explains when reshaping-based algorithms for computing tensor rank decompositions may be expected to recover the decomposition. Specializing the analysis to symmetric tensors or forms reveals that the reshaped Kruskal criterion may even be effective up to the smallest typical rank for symmetric tensors of small dimension as well as for binary forms of degree at least three. We extend these results to $4 \times 4 \times 4 \times 4$ symmetric tensors by analyzing the Hilbert function, resulting in a criterion for symmetric identifiability that is effective up to symmetric rank 8 , which is optimal.
\end{abstract}

Key words. tensor rank decomposition, Waring decomposition, effective identifiability, reshaped Kruskal criterion, Hilbert function

AMS subject classifications. 15A69, 15A72, 14N20, 14N05, 14Q15, 14Q20

1. Introduction. A tensor rank decomposition expresses a tensor $\mathfrak{A} \in \mathbb{F}^{n_{1}} \otimes$ $\mathbb{F}^{n_{2}} \otimes \cdots \otimes \mathbb{F}^{n_{d}}$ as a linear combination of rank-1 tensors, as follows:

$$
\mathfrak{A}=\sum_{i=1}^{r} \mathbf{a}_{i}^{1} \otimes \mathbf{a}_{i}^{2} \otimes \cdots \otimes \mathbf{a}_{i}^{d}
$$

where $\mathbf{a}_{i}^{k} \in \mathbb{F}^{n_{k}}$, and $\mathbb{F}$ is either the real field $\mathbb{R}$ or complex field $\mathbb{C}$. When $r$ is minimal in the above expression, then it is called the rank of $\mathfrak{A}$. A key property of the tensor rank decomposition is its generic identifiability [15,21,28]. This means that the expression (1) is unique up to a permutation of the summands and scaling of the vectors on a dense, open subset of the set of tensors admitting an expression as in (1). This uniqueness property renders it useful in several applications. For instance, in chemometrics, decomposition (1) arises in the simultaneous spectral analysis of unknown mixtures of fluorophores, where the tensor rank decomposition of the corresponding tensor reveals the emission-excitation matrices of the individual chemical molecules in the mixtures, hence allowing a trained chemist to identify the fluorophores [5].

Another application of tensor decompositions is parameter identification in statistical models with hidden variables, such as principal component analysis (or blind source separation), exchangeable single topic models and hidden Markov models. Such applications were recently surveyed in a tensor-based framework in [3]. The key in

\footnotetext{
*Submitted to the editors.

Funding: The first and second author are members of the Italian GNSAGA-INDAM. The third author was supported by a Postdoctoral Fellowship of the Research Foundation-Flanders (FWO).

$\dagger$ Dipartimento di Ingegneria dell'Informazione e Scienze Matematiche, Università di Siena, Italy (luca.chiantini@unisi.it).

¥Dipartimento di Matematica e Informatica "Ulisse Dini", Università di Firenze, Italy (ottavian@math.unifi.it).

$\S$ KU Leuven, Department of Computer Science, Belgium (nick.vannieuwenhoven@cs.kuleuven.be).
} 
these applications consists of recovering the unknown parameters by computing a Waring decomposition of a higher-order moment tensor constructed from the known samples. In other words, one seeks a decomposition

$$
\mathfrak{A}=\sum_{i=1}^{r} \lambda_{i} \mathbf{a}_{i} \otimes \cdots \otimes \mathbf{a}_{i}=\sum_{i=1}^{r} \lambda_{i} \mathbf{a}_{i}^{\otimes d},
$$

where $\mathbf{a}_{i} \in \mathbb{F}^{n}$ and $\lambda_{i} \in \mathbb{F}$ for all $i=1, \ldots, r$. Note that $\mathfrak{A}$ is a symmetric tensor in this case. If $r$ is minimal, then $r$ is called the Waring or symmetric rank of $\mathfrak{A}$. Uniqueness of Waring decompositions is again the key for ensuring that the recovered parameters of the model are unique and interpretable. Generic identifiability of complex Waring decompositions of subgeneric rank for nearly all tensor spaces was proved in [22].

We address in this paper the problem of specific identifiability: given a tensor rank decomposition of length $r$ in the space $\mathbb{F}^{n_{1}} \otimes \mathbb{F}^{n_{2}} \otimes \cdots \otimes \mathbb{F}^{n_{d}}$, prove that it is unique. Let $\mathcal{S}$ denote the variety of rank-1 tensors in this space. As it is conjectured that the generic ${ }^{1}$ tensor of subtypical ${ }^{2}$ rank $r$, i.e.,

$$
r<\bar{r}_{\mathcal{S}}=\frac{n_{1} n_{2} \cdots n_{d}}{n_{1}+n_{2}+\cdots+n_{d}-d+1}
$$

has a unique decomposition, provided it is not one of the exceptional cases listed in [21, Theorem 1.1], we believe that any practical criterion for specific identifiability must be more informative than the following naive Monte Carlo algorithm:

S1. If the number of terms in the given tensor decomposition is less than $\bar{r}_{\mathcal{S}}$, then claim "Identifiable," otherwise claim "Not identifiable."

This simple algorithm has a $100 \%$ probability of returning a correct result if one samples decompositions of length $r$ from any probability distribution whose support is not contained in the Zariski-closed locus where $r$-identifiability fails (assuming generic $r$-identifiability; see Section 3). It also has a $0 \%$ chance of returning an incorrect answer - it can be wrong, e.g., if the unidentifiable tensor $\mathbf{a} \otimes \mathbf{a} \otimes \mathbf{a}+\mathbf{b} \otimes \mathbf{b} \otimes \mathbf{a}$ is presented as input, but the probability of sampling these tensors is zero. Deterministic algorithms for specific $r$-identifiability, e.g., [21,26,36, 38, 50,53], merit consideration, but only if they are what we propose to call effective: if they can prove identifiability on a dense, open subset of the set of tensors admitting decomposition (1). A deterministic criterion is thus effective if its conditions are satisfied generically; that is, if the same criterion also proves generic identifiability. Kruskal's well-known criterion for $r$-identifiability is deterministic: it is a sufficient condition for uniqueness. If the criterion is not satisfied, the outcome of the test is inconclusive. Effective criteria are allowed to have such inconclusive outcomes provided that they do not form a Euclidean-open set. It will not surprise the experts that Kruskal's criterion [38] is effective. Domanov and De Lathauwer [28] recently proved that some of their criteria for third-order tensors from [26] are effective. Presently, only a few effective criteria for specific $r$-identifiability of tensors of higher order, i.e., $d \geq 4$, are -informallyknown, notably the generalization of Kruskal's criterion to higher-order tensors due to Sidiropoulos and Bro [50].

In private communication, I. Domanov remarked that "in practice, when one wants to check that the [tensor rank decomposition] of a tensor of order higher than 3

\footnotetext{
${ }^{1}$ We call $p \in S$ "generic" with respect to some property in the set $S$, if the property fails to hold at most for the elements in a strict subvariety of $S$.

${ }^{2}$ Recall that the smallest typical rank over $\mathbb{R}$ coincides with the generic rank over $\mathbb{C}[13]$, hence the term subtypical through all this paper coincides with the term subgeneric used in [22].
} 
is unique, [one] just reshapes the tensor into a third-order tensor and then applies the classical Kruskal result [...]. The reduction to the third-order case is quite standard and well-known;" indeed this idea appears also in [18,40,46,50,51]. Formally, it can be stated as follows. Let $\mathbf{h} \cup \mathbf{k} \cup \mathbf{l}=\{1,2, \ldots, d\}$ be a partition where $\mathbf{h}, \mathbf{k}$ and $\mathbf{l}$ have cardinalities $d_{1}, d_{2}$ and $d_{3}$ respectively. Let $\mathcal{S}=\operatorname{Seg}\left(\mathbb{F}^{n_{1}} \times \cdots \times \mathbb{F}^{n_{d}}\right)$ be the variety of rank-1 tensors in $\mathbb{F}^{n_{1}} \otimes \cdots \otimes \mathbb{F}^{n_{d}}$, and let $\mathcal{S}_{\mathbf{h}, \mathbf{k}, \mathbf{l}}=\operatorname{Seg}\left(\mathbb{F}^{n_{h_{1}} \cdots n_{h_{d_{1}}}} \times \mathbb{F}^{n_{k_{1}} \cdots n_{k_{d_{2}}}} \times\right.$ $\mathbb{F}^{n_{l_{1}} \cdots n_{l_{2}}}$ ) be the variety of rank-1 tensors in the reshaped tensor space. We may consider the natural inclusion $\mathcal{S} \hookrightarrow \mathcal{S}_{\mathbf{h}, \mathbf{k}, \mathbf{l}}$ and then apply a criterion for specific $r$ identifiability with respect to $\mathcal{S}_{\mathbf{h}, \mathbf{k}, \mathbf{l}}$. If this criterion certifies $r$-identifiability, then it entails $r$-identifiability with respect to $\mathcal{S}$ as well. While this idea is valid, applying an effective criterion for third-order tensors to reshaped higher-order tensors does not suffice for concluding that it is also an effective criterion for higher-order tensors. Indeed, since $\mathcal{S}$ has dimension strictly less than $\mathcal{S}_{\mathbf{h}, \mathbf{k}, \mathbf{l}}$ one expects that the set of rank- $r$ tensors in $\mathbb{F}^{n_{1}} \otimes \cdots \otimes \mathbb{F}^{n_{d}}$ constitutes a Zariski-closed subset of the rank- $r$ tensors in the reshaped tensor space. As a result, the effective criterion for $\mathcal{S}_{\mathbf{h}, \mathbf{k}, \mathbf{1}}$ might thus never apply to the elements of $\mathcal{S} \hookrightarrow \mathcal{S}_{\mathbf{h}, \mathbf{k}, \mathbf{l}}$. This observation was the impetus for the present work and the reason why our results will always be presented in the general setting.

Our first main result, proved in Section 4, can be stated informally as follows.

THEOREM 1. Kruskal's criterion applied to a reshaped rank-r tensor is an effective criterion for specific $r$-identifiability.

The reshaped Kruskal criterion as well as the criteria in [26, 28, 36] applied to a reshaped tensor can all be considered as state-of-the-art results in specific identifiability. Nevertheless, combining reshaping with a criterion for lower-order identifiability may not be expected to prove specific identifiability up to the (nearly) optimal value $\bar{r}_{\mathcal{S}}-1$. Indeed, consider any partition $\mathbf{h}_{1} \cup \cdots \cup \mathbf{h}_{t}=\{1,2, \ldots, d\}$ with $t<d$. Then,

$$
\bar{r}_{\mathcal{S}_{\mathbf{h}_{1}, \ldots, \mathbf{h}_{t}}}=\frac{n_{1} n_{2} \cdots n_{d}}{1+\sum_{k=1}^{t}\left(-1+\prod_{\ell \in \mathbf{h}_{k}} n_{\ell}\right)} \leq \frac{n_{1} n_{2} \cdots n_{d}}{n_{1}+n_{2}+\cdots+n_{d}-d+1}=\bar{r}_{\mathcal{S}},
$$

where typically the integers $n_{i} \geq 2$ are such that a strict inequality occurs.

In the second half of the paper, the reshaped Kruskal criterion is considered for symmetric tensors. Remarkably, in 6 cases of small dimension as well as for binary forms this criterion is completely effective: the criterion is effective in the entire range where generic $r$-identifiability holds. Algorithm 3 from [45] is completely effective in two additional cases. We show that an analysis of the Hilbert function yields another case, namely symmetric tensors in $\mathbb{F}^{4} \otimes \mathbb{F}^{4} \otimes \mathbb{F}^{4} \otimes \mathbb{F}^{4}$. The second main result, which is proved in Section 6 , can be stated as follows.

THEOREM 2. Let $S^{d} \mathbb{F}^{n}$ be the linear subspace of symmetric tensors in $\mathbb{F}^{n} \otimes \cdots \otimes$ $\mathbb{F}^{n}$. Then, there exist completely effective criteria for specific symmetric identifiability of symmetric tensors in the following cases: $S^{3} \mathbb{F}^{n}$ with $n=3,4,5,6, S^{k} \mathbb{F}^{2}$ for $k \geq 3$, $S^{k} \mathbb{F}^{3}$ with $k=3,4,5,6,7$, and $S^{4} \mathbb{F}^{4}$.

These are the only cases we know of where effective criteria for specific identifiability exist that can be applied up to the bound for generic identifiability.

The outline of the remainder of this paper is as follows. In the next section, some preliminary material is recalled. The known results about generic identifiability are presented in Section 3. We analyze the reshaped Kruskal criterion in Section 4: we prove that it is an effective criterion and present a heuristic for choosing a good reshaping. Section 5 presents the variant of the reshaped Kruskal criterion for symmetric tensors, proving most cases of the second main result. It also explains 
how analyzing the Hilbert function can lead to results about specific identifiability for symmetric tensors. These insights culminate in Section 6 , where we complete the proof the second main result, then provide an algorithm implementing that effective criterion, and finally present some concrete examples. In Section 7, we explain when reshaping-based algorithms for computing tensor rank decompositions will recover the decomposition. Section 8 presents our main conclusions.

Notation. Varieties are typeset in a calligraphic font, tensors in a fraktur font, matrices in upper case, and vectors in boldface lower case. The field $\mathbb{F}$ denotes either $\mathbb{R}$ or $\mathbb{C}$, and $\mathbb{F}_{0}=\mathbb{F} \backslash\{0\}$. Projectivization is denoted by $\mathbb{P} . \quad V$ denotes a finitedimensional vector space over the field $\mathbb{F}$. The matrix transpose and conjugate transpose are denoted by $\cdot^{T}$ and ${ }^{H}$ respectively. The Khatri-Rao product of $A \in \mathbb{F}^{m \times r}$ and $B \in \mathbb{F}^{n \times r}$ is $A \odot B=\left[\begin{array}{llll}\mathbf{a}_{1} \otimes \mathbf{b}_{1} & \mathbf{a}_{2} \otimes \mathbf{b}_{2} & \cdots & \mathbf{a}_{r} \otimes \mathbf{b}_{r}\end{array}\right]$. A set partition is denoted by $S_{1} \sqcup \cdots \sqcup S_{k}=\{1, \ldots, m\}$. If $\mathcal{X}$ is a variety, then $\mathcal{X}_{0}$ is defined as $\mathcal{X}$ minus the zero element. The affine cone over a projective variety $\mathcal{X} \subset \mathbb{P F}^{n}$ is $\widehat{\mathcal{X}}:=\{\alpha x \mid x \in \mathcal{X}, \alpha \in \mathbb{F}\}$. The Segre variety $\operatorname{Seg}\left(\mathbb{P F}^{n_{1}} \times \cdots \times \mathbb{P F}^{n_{d}}\right) \subset \mathbb{P}\left(\mathbb{F}^{n_{1}} \otimes \cdots \otimes \mathbb{F}^{n_{d}}\right)$ is denoted by $\mathcal{S}$, and the Veronese variety $v_{d}\left(\mathbb{P F}^{n}\right) \subset \mathbb{P} S^{d} \mathbb{F}^{n}$ is denoted by $\mathcal{V}$. The projective dimension of the Segre variety $\mathcal{S}$ is denoted by $\Sigma=\sum_{k=1}^{d}\left(n_{k}-1\right)$. The dimension of $\mathbb{F}^{n_{1}} \otimes \cdots \otimes \mathbb{F}^{n_{d}}$ is $\Pi=\prod_{k=1}^{d} n_{k}$, and the dimension of $S^{d} \mathbb{F}^{n}$ is $\Gamma=\left(\begin{array}{c}n-1+d \\ d\end{array}\right)$.

2. Preliminaries. We recall some terminology from algebraic geometry; the reader is referred to Landsberg [40] for a more detailed discussion.

2.1. Segre and Veronese varieties. The set of rank- 1 tensors in the projective space $\mathbb{P}\left(\mathbb{F}^{n_{1}} \otimes \mathbb{F}^{n_{2}} \otimes \cdots \otimes \mathbb{F}^{n_{d}}\right)$ is a projective variety, called the Segre variety. It is the image of the Segre map

$$
\begin{aligned}
& \text { Seg }: \mathbb{P F}^{n_{1}} \times \mathbb{P F}^{n_{2}} \times \cdots \times \mathbb{P F}^{n_{d}} \rightarrow \mathbb{P}\left(\mathbb{F}^{n_{1}} \otimes \mathbb{F}^{n_{2}} \otimes \cdots \otimes \mathbb{F}^{n_{d}}\right) \cong \mathbb{P F}^{n_{1} n_{2} \cdots n_{d}} \\
&\left(\left[\mathbf{a}^{1}\right],\left[\mathbf{a}^{2}\right], \ldots,\left[\mathbf{a}^{d}\right]\right) \mapsto\left[\mathbf{a}^{1} \otimes \mathbf{a}^{2} \otimes \cdots \otimes \mathbf{a}^{d}\right]
\end{aligned}
$$

where $[\mathbf{a}]=\left\{\lambda \mathbf{a} \mid \lambda \in \mathbb{F}_{0}\right\}$ is the equivalence class of $\mathbf{a} \in \mathbb{F}^{n} \backslash\{0\}$. The Segre variety will be denoted by $\mathcal{S}$. Its dimension is $\Sigma=\operatorname{dim} \mathcal{S}=\sum_{k=1}^{d}\left(n_{k}-1\right)$.

The symmetric rank-1 tensors in $\mathbb{P}\left(\mathbb{F}^{n} \otimes \cdots \otimes \mathbb{F}^{n}\right)$ constitute an algebraic variety that is called the Veronese variety. It is obtained as the image of

$$
\text { Ver }: \mathbb{P} \mathbb{F}^{n} \rightarrow \mathbb{P}\left(\mathbb{F}^{n} \otimes \cdots \otimes \mathbb{F}^{n}\right),[\mathbf{a}] \mapsto\left[\mathbf{a}^{\otimes d}\right] \text {. }
$$

The Veronese variety will be denoted by $\mathcal{V}$, and its dimension is $\operatorname{dim} \mathcal{V}=n-1$. The span of the image of the Veronese map is the projectivization of the linear subspace of $\mathbb{F}^{n} \otimes \cdots \otimes \mathbb{F}^{n}$ consisting of the symmetric tensors, namely $\left\{\mathfrak{A} \mid a_{i_{1}, i_{2}, \ldots, i_{d}}=\right.$ $\left.a_{i_{\sigma_{1}}, i_{\sigma_{2}}, \ldots, i_{\sigma_{d}}}, \forall \sigma \in S\right\}$, where $S$ is the set of all permutations of $\{1,2, \ldots, d\}$. This space is isomorphic to the $d$ th symmetric power of $\mathbb{F}^{n}$, i.e., $S^{d} \mathbb{F}^{n}=\mathbb{F}\left(\begin{array}{c}n+d \\ d\end{array}\right)$, as can be understood from

$$
v_{d}: \mathbb{P F}^{n} \rightarrow \mathbb{P}\left(S^{d} \mathbb{F}^{n}\right),[\mathbf{a}] \mapsto\left[\mathbf{a}^{\circ d}\right]=\left[a_{i_{1}} a_{i_{2}} \cdots a_{i_{d}}\right]_{1 \leq i_{1} \leq i_{2} \leq \cdots \leq i_{d} \leq n},
$$

where $\mathbf{a}^{\text {od }}$ is called the $d$ th symmetric power of $\mathbf{a}$. The homogeneous polynomials of degree $d$ in $n$ variables correspond bijectively with $S^{d} \mathbb{F}^{n}[24,35]$. Therefore, the elements of $\mathbb{P}\left(S^{d} \mathbb{F}^{n}\right)$ are often called $d$-forms or simply forms when the degree is clear.

2.2. Secants of varieties. Define for a smooth irreducible projective variety $\mathcal{X} \subset \mathbb{P} V$ that is not contained in a hyperplane, such as a Segre or Veronese variety, 
the abstract $r$-secant variety $\operatorname{Abs} \sigma_{r}(\mathcal{X})$ as the closure in the Euclidean topology of

$$
\operatorname{Abs} \sigma_{r}^{0}(\mathcal{X}):=\left\{\left(\left(p_{1}, p_{2}, \ldots, p_{r}\right), p\right) \mid p \in\left\langle p_{1}, p_{2}, \ldots, p_{r}\right\rangle, p_{i} \in \mathcal{X}\right\} \subset \mathcal{X}^{\times r} \times \mathbb{P} V,
$$

Let the image of the projection of $\operatorname{Abs} \sigma_{r}^{0}(\mathcal{X}) \subset \mathcal{X}^{\times r} \times \mathbb{P} V$ onto the last factor be denoted by $\sigma_{r}^{0}(\mathcal{X})$. Then, the $r$-secant semi-algebraic set of $\mathcal{X}$, denoted by $\sigma_{r}(\mathcal{X})$, is defined as the closure in the Euclidean topology of $\sigma_{r}^{0}(\mathcal{X})$. It is an irreducible semialgebraic set because of the Tarski-Seidenberg theorem [16]. For $\mathbb{F}=\mathbb{C}$, the Zariskiclosure coincides with the Euclidean closure and $\sigma_{r}(\mathcal{X})$ is a projective variety [40]. It follows that $\operatorname{dim} \sigma_{r}(\mathcal{X}) \leq \min \{r(\operatorname{dim} \mathcal{X}+1), \operatorname{dim} V\}-1$. If the inequality is strict then we say that $\mathcal{X}$ has an $r$-defective secant semi-algebraic set. If $\mathcal{X}$ has no defective secant semi-algebraic sets then it is called a nondefective semi-algebraic set. The $\mathcal{X}$ rank of a point $p \in \mathbb{P} V$ is defined as the least $r$ for which $p=\left[p_{1}+\cdots+p_{r}\right]$ with $p_{i} \in \widehat{\mathcal{X}}$; we will write $\operatorname{rank}(p)=r$.

For a nondefective variety $\mathcal{X} \subset \mathbb{P} V$ not contained in a hyperplane, we define the expected smallest typical rank of $\mathcal{X}$ as the least integer larger than or equal to

$$
\bar{r}_{\mathcal{X}}=\frac{\operatorname{dim} V}{1+\operatorname{dim} \mathcal{X}}
$$

namely $\left\lceil\bar{r}_{\mathcal{X}}\right\rceil$. With this definition, the expected smallest typical rank of a nondefective complex Segre variety $\mathcal{S}_{\mathbb{C}} \subset \mathbb{P} V$ coincides with the value of $r$ for which $\sigma_{r}\left(\mathcal{S}_{\mathbb{C}}\right)=\mathbb{P} V$, so that $\sigma_{\left\lceil\bar{r}_{\mathcal{C}}\right\rceil}^{0}\left(\mathcal{S}_{\mathbb{C}}\right)$ is a Euclidean-dense subset of $\mathbb{P} V$. In the case of a nondefective real Segre variety $\mathcal{S}_{\mathbb{R}} \subset \mathbb{P V}$, the expected smallest typical rank as defined above coincides with the smallest typical rank; a rank $r$ is typical if the affine cone over $\sigma_{r}^{0}\left(\mathcal{S}_{\mathbb{R}}\right) \backslash \sigma_{r-1}^{0}\left(\mathcal{S}_{\mathbb{R}}\right) \subset \mathbb{P} V$ is open in the Euclidean topology on $V$. Note that $\bar{r}_{\mathcal{S}_{\mathbb{R}}}=\bar{r}_{\mathcal{S}_{\mathbb{C}}}$ and that in $\mathbb{F}=\mathbb{C}$ there is only one typical rank, which is hence the generic rank; see [13]. For a nondefective variety $\mathcal{X}$, the generic element $[p] \in \sigma_{r}(\mathcal{X})$ with $r \leq \bar{r}_{\mathcal{X}}$ has $\operatorname{rank}([p])=\operatorname{rank}(p)=r$, and, furthermore, it admits finitely many decompositions of the form $p=p_{1}+\cdots+p_{r}$ with $p_{i} \in \widehat{\mathcal{X}}$; see [40]. For $r>\bar{r}_{\mathcal{X}}$, it follows from a dimension count that the generic $p \in \sigma_{r}(\mathcal{X})$ admits infinitely many decompositions of the foregoing type, because the generic fiber of the projection map $\operatorname{Abs} \sigma_{r}(\mathcal{X}) \rightarrow$ $\sigma_{r}(\mathcal{X})$ has dimension $r(\operatorname{dim} \mathcal{X}+1)-\operatorname{dim} V$. This observation also holds for $r$-defective secant semi-algebraic sets where the generic fiber has a dimension equal to the defect.

2.3. Inclusions, projections, and flattenings. Let $\mathbf{h} \sqcup \mathbf{k}=\{1,2, \ldots, d\}$ with $\mathbf{h}$ and $\mathbf{k}$ of cardinality $s>0$ and $t>0$ respectively. Several criteria for identifiability rely on the natural inclusion into two-factor Segre varieties, namely

$\mathcal{S}=\operatorname{Seg}\left(\mathbb{P F}^{n_{1}} \times \cdots \times \mathbb{P F}^{n_{d}}\right) \hookrightarrow \operatorname{Seg}\left(\mathbb{P}\left(\mathbb{F}^{n_{h_{1}}} \otimes \cdots \otimes \mathbb{F}^{n_{h_{s}}}\right) \times \mathbb{P}\left(\mathbb{F}^{n_{k_{1}}} \otimes \cdots \otimes \mathbb{F}^{n_{k_{t}}}\right)\right)=\mathcal{S}_{\mathbf{h}, \mathbf{k}}$,

or the inclusion into three-factor Segre varieties, which can be defined analogously and for which we employ the notation $\mathcal{S}_{\mathbf{h}, \mathbf{k}, \mathbf{l}}$, where $\mathbf{h} \sqcup \mathbf{k} \sqcup \mathbf{l}=\{1,2, \ldots, d\}$.

Let $\mathbf{h} \subset\{1,2, \ldots, d\}$ be of cardinality $s>0$. Define the projections

$$
\begin{aligned}
\pi_{\mathbf{h}}: \mathcal{S}=\operatorname{Seg}\left(\mathbb{P F}^{n_{1}} \times \mathbb{P F}^{n_{2}} \times \cdots \times \mathbb{P F}^{n_{d}}\right) & \rightarrow \operatorname{Seg}\left(\mathbb{P F}^{n_{h_{1}}} \times \cdots \times \mathbb{P F}^{n_{h_{s}}}\right)=\mathcal{S}_{\mathbf{h}} \\
{\left[\mathbf{a}_{1} \otimes \mathbf{a}_{2} \otimes \cdots \otimes \mathbf{a}_{d}\right] } & \mapsto\left[\mathbf{a}_{h_{1}} \otimes \mathbf{a}_{h_{2}} \otimes \cdots \otimes \mathbf{a}_{h_{s}}\right] .
\end{aligned}
$$

We will abuse notation by writing $\pi_{\mathbf{h}}(p)=\mathbf{a}_{h_{1}} \otimes \cdots \otimes \mathbf{a}_{h_{s}}$ if $p=\mathbf{a}_{1} \otimes \cdots \otimes \mathbf{a}_{d} \in \widehat{\mathcal{S}}$.

Flattenings are defined as follows. Let $\mathbf{h} \sqcup \mathbf{k}=\{1,2, \ldots, d\}$ with $\mathbf{h}$ and $\mathbf{k}$ of cardinality $s \geq 0$ and $t \geq 0$ respectively. Then, the $(\mathbf{h}, \mathbf{k})$-flattening, or simply $\mathbf{h}$ flattening, of $p \in \widehat{\mathcal{S}}$ is the natural inclusion of $p \in \widehat{\mathcal{S}}$ into $\widehat{\mathcal{S}}_{\mathbf{h}, \mathbf{k}}$ :

$$
p_{(\mathbf{h})}=\pi_{\mathbf{h}}(p) \pi_{\mathbf{k}}(p)^{T} \in \widehat{\mathcal{S}}_{\mathbf{h}, \mathbf{k}} \subset \mathbb{F}^{n_{h_{1}} \cdots n_{h_{s}}} \otimes \mathbb{F}^{n_{k_{1}} \cdots n_{k_{t}}} \cong \mathbb{F}^{n_{h_{1}} \cdots n_{h_{s}} \times n_{k_{1}} \cdots n_{k_{t}}} .
$$


By convention $\pi_{\emptyset}(p)=1$. A $(\mathbf{h}, \mathbf{k})$-flattening of a rank- $r$ tensor $p=p_{1}+\cdots+p_{r}$ with $p_{i} \in \widehat{\mathcal{S}}$ is then defined as $p_{(\mathbf{h})}=\left(p_{1}\right)_{(\mathbf{h})}+\cdots+\left(p_{r}\right)_{(\mathbf{h})}$.

3. Generic identifiability of tensors and forms. Let $\mathcal{X}$ be an irreducible, nondegenerate algebraic $\mathbb{F}$-variety that contains at least one smooth real point if $\mathbb{F}=\mathbb{R}$. By convention, we call a rank- $r$ decomposition $p=p_{1}+\cdots+p_{r}, p_{i} \in \widehat{\mathcal{X}}$, distinct from another decomposition $p=q_{1}+\cdots+q_{r}, q_{i} \in \widehat{\mathcal{X}}$, if there does not exist a permutation $\sigma$ of $\{1,2, \ldots, r\}$ such that $p_{i}=q_{\sigma_{i}}$ for all $i$. We say that $\mathcal{X}$ is generically $r$-identifiable if the set of tensors with multiple distinct complex decompositions in $\sigma_{r}(\mathcal{X})$ is contained in a proper Zariski-closed subset of $\sigma_{r}(\mathcal{X})$. This concept is meaningful only when $r$ is subtypical, i.e., $r<\bar{r}_{\mathcal{X}}$, or if $r=\bar{r}_{\mathcal{X}}$ is an integer, i.e., when the tensor space is perfect. The generic tensor $p \in \sigma_{r}(\mathcal{X})$ cannot admit a finite number of decompositions of length $r$ if $r>\bar{r}_{\mathcal{X}}$ because of the dimension argument mentioned in Section 2.2.

Complex generic $r$-identifiability of the Waring decomposition (2) has been completely characterized in the literature.

Theorem 3. Let $d \geq 3$, and let $\mathbb{F}=\mathbb{C}$ or $\mathbb{R}$. Let $\mathcal{V}_{d, n}^{\mathbb{E}}$ be the $d$ th Veronese embedding of $\mathbb{F}^{n}$ in $\mathbb{P} S^{d} \mathbb{F}^{n}$. Then, $\mathcal{V}_{d, n}^{\mathbb{F}}$ is generically r-identifiable for all strictly subtypical ranks $r<n^{-1}\left(\begin{array}{c}n-1+d \\ d\end{array}\right)$, unless it is one of the following cases:

1. $\mathcal{V}_{3,6}^{\mathbb{F}}$ and $r=9$;

2. $\mathcal{V}_{4,4}^{\mathbb{E}}$ and $r=8$; or

3. $\mathcal{V}_{6,3}^{\mathbb{F}}$ and $r=9$.

The generic tensor has 2 distinct complex decompositions in these exceptional cases.

Proof. This theorem was proved for $\mathbb{F}=\mathbb{C}$ in [22]. It can be extended to $\mathbb{F}=\mathbb{R}$ by invoking the beautiful result of Qi, Comon, and Lim [47, Lemma 28].

Remark 4. Arguing from the abstract $r$-secant variety, the results of [47, Section $5]$ entail that $\sigma_{r}^{0}\left(\mathcal{S}_{\mathbb{R}}\right)$ is not contained in the singular locus of $\sigma_{r}\left(\mathcal{S}_{\mathbb{C}}\right)$, which has codimension at least 1 in $\sigma_{r}\left(\mathcal{S}_{\mathbb{C}}\right)$. Let the Nash stratification [16] of the semi-algebraic set $\sigma_{r}^{0}\left(\mathcal{S}_{\mathbb{R}}\right)$ be given by $\sigma_{r}^{0}\left(\mathcal{S}_{\mathbb{R}}\right)=\cup_{i=1}^{k} \mathcal{N}_{i}$, with $\mathcal{N}_{i}$ a Nash manifold and $\mathcal{N}_{i} \cap \mathcal{N}_{j}=\emptyset$ if $i \neq j$. Let $\mathcal{N}_{i}$ be an arbitrary Nash manifold not contained in $\operatorname{Sing}\left(\sigma_{r}\left(\mathcal{S}_{\mathbb{C}}\right)\right)$, and let $[p] \in \mathcal{N}_{i} \backslash \operatorname{Sing}\left(\sigma_{r}\left(\mathcal{S}_{\mathbb{C}}\right)\right)$, so it has a decomposition $p=p_{1}+\cdots+p_{r}$, where $p_{i} \in \widehat{\mathcal{S}}_{\mathbb{R}}$. Since $p$ is smooth in $\sigma_{r}\left(\mathcal{S}_{\mathbb{C}}\right)$ its tangent space is $\mathrm{T}_{p} \sigma_{r}\left(\mathcal{S}_{\mathbb{C}}\right)=\left\langle\mathrm{T}_{p_{1}} \mathcal{S}_{\mathbb{C}}, \ldots, \mathrm{T}_{p_{r}} \mathcal{S}_{\mathbb{C}}\right\rangle=$ $\left\langle\mathrm{T}_{p_{1}} \mathcal{S}_{\mathbb{R}} \otimes \mathbb{C}, \ldots, \mathrm{T}_{p_{r}} \mathcal{S}_{\mathbb{R}} \otimes \mathbb{C}\right\rangle=\mathrm{T}_{p} \sigma_{r}\left(\mathcal{S}_{\mathbb{R}}\right) \otimes \mathbb{C}$ by Terracini's lemma. Hence, $\operatorname{dim}_{\mathbb{R}} \mathcal{N}_{i}=$ $\operatorname{dim}_{\mathbb{C}} \sigma_{r}\left(\mathcal{S}_{\mathbb{C}}\right){ }^{3} \quad$ Let $\mathcal{U} \subset \sigma_{r}\left(\mathcal{S}_{\mathbb{C}}\right)$ be the locus where complex $r$-identifiability fails, whose Zariski-closure is of codimension at least 1 by Theorem 1.1 of [22]. Then $\mathcal{N}_{i} \cap \mathcal{U}$ is contained in a proper Zariski-closed set of $\mathcal{N}_{i} \subset \sigma_{r}\left(\mathcal{S}_{\mathbb{C}}\right)$, namely $\overline{\mathcal{U}}$, proving that $\sigma_{r}\left(\mathcal{S}_{\mathbb{R}}\right)$ is generically $r$-identifiable if $\sigma_{r}\left(\mathcal{S}_{\mathbb{C}}\right)$ is generically $r$-identifiable.

If complex $r$-identifiability fails on a Zariski-open set, then there is a Euclideanopen set of real decomposition of real rank $r$ admitting multiple complex decompositions, but we do not presently know how many of these are real. We leave this as an open problem warranting further research. Recently, some interesting progress in this direction was made in [4].

This theorem completely settles the question concerning the number of complex Waring decompositions (2) of the generic symmetric tensor of strictly subtypical rank $r<\bar{r}_{\mathcal{V}}$ : aside from the listed exceptions, it is one. In the perfect case where $\bar{r}_{\mathcal{V}}$ is an integer and $\mathbb{F}=\mathbb{C}$, the following remarkable result was recently proved.

\footnotetext{
${ }^{3}$ Note that all $\mathcal{N}_{i}$ whose dimension is less than $\operatorname{dim}_{\mathbb{C}} \sigma_{r}\left(\mathcal{S}_{\mathbb{C}}\right)$ are contained in $\operatorname{Sing}\left(\sigma_{r}\left(\mathcal{S}_{\mathbb{C}}\right)\right)$.
} 
Theorem 5 (Galuppi and Mella [29]). Let $d \geq 3$. Let $\mathcal{V}_{d, n}$ be the $d$ th Veronese embedding of $\mathbb{C}^{n}$ in $\mathbb{P} S^{d} \mathbb{C}^{n}$ and assume that $\bar{r}_{\mathcal{V}}=n^{-1}\left(\begin{array}{c}n-1+d \\ d\end{array}\right)$ is an integer. $\mathcal{V}_{d, n}$ is generically $\bar{r}_{\mathcal{V}_{d, n}}$-identifiable if and only if it is either $\mathcal{V}_{2 k+1,2}$ with $k \geq 1, \mathcal{V}_{3,4}$ or $\mathcal{V}_{5,3}$.

In summary we can state that the generic symmetric tensor of rank $r$ in all but a few tensor spaces $S^{d} \mathbb{F}^{n}$ admits a unique Waring decomposition over $\mathbb{F}$ if $r$ is subtypical, while it is expected to admit several decompositions if $r \geq \bar{r} \mathcal{V}_{d, n}$.

The theory of generic identifiability of the Segre variety is less developed than the Veronese variety. Because of the corroborating evidence in $[15,20,21,28,33,54]$, the following conjectures are believed to be true.

Conjecture 6. Let $d \geq 3$, and let $n_{1} \geq \cdots \geq n_{d} \geq 2$. Let $\mathcal{S}_{\mathbb{F}}=\operatorname{Seg}\left(\mathbb{P F}^{n_{1}} \times \cdots \times\right.$ $\mathbb{P F}^{n_{d}}$ ) be the Segre variety in $\mathbb{P}\left(\mathbb{F}^{n_{1}} \otimes \cdots \otimes \mathbb{F}^{n_{d}}\right)$. Then, $\mathcal{S}_{\mathbb{F}}$ is generically $r$-identifiable for all strictly subtypical ranks $r<\bar{r}_{\mathcal{S}_{\mathbb{F}}}$, unless it is one of the following cases:

1. $n_{1}>\prod_{k=2}^{d} n_{k}-\sum_{k=2}^{d}\left(n_{k}-1\right)$ and $r \geq \prod_{k=2}^{d} n_{k}-\sum_{k=2}^{d}\left(n_{k}-1\right)$;

2. $\mathcal{S}=\operatorname{Seg}\left(\mathbb{P F}^{4} \times \mathbb{P F}^{4} \times \mathbb{P F}^{3}\right)$ and $r=5$;

3. $\mathcal{S}=\operatorname{Seg}\left(\mathbb{P F}^{n} \times \mathbb{P F}^{n} \times \mathbb{P F}^{2} \times \mathbb{P F}^{2}\right)$ and $r=2 n-1$;

4. $\mathcal{S}=\operatorname{Seg}\left(\mathbb{P F}^{4} \times \mathbb{P F}^{4} \times \mathbb{P F}^{4}\right)$ and $r=6$;

5. $\mathcal{S}=\operatorname{Seg}\left(\mathbb{P F}^{6} \times \mathbb{P F}^{6} \times \mathbb{P F}^{3}\right)$ and $r=8$; or

6. $\mathcal{S}=\operatorname{Seg}\left(\mathbb{P F}^{2} \times \mathbb{P F}^{2} \times \mathbb{P F}^{2} \times \mathbb{P F}^{2} \times \mathbb{P F}^{2}\right)$ and $r=5$;

The first three cases generically admit infinitely many decompositions [1, 15]. Case (4) generically admits 2 complex decompositions [20], case (5) is expected ${ }^{4}$ to generically admit 6 complex decompositions, and case (6) admits generically 2 complex decompositions [14].

Remark 7 . The conjecture was initially stated for $\mathbb{F}=\mathbb{C}$ in $[15,21]$. Theorem 1.1 of [21], which proves Conjecture 6 for all $n_{1} n_{2} \cdots n_{d} \leq 15000$ with $\mathbb{F}=\mathbb{C}$, can be extended to $\mathbb{F}=\mathbb{R}$ as in Remark 4 by invoking Qi, Comon, and Lim's analysis [47].

Conjecture 8 (Hauenstein, Oeding, Ottaviani, and Sommese [33]). Let $d \geq 3$, and let $n_{1} \geq n_{2} \geq \cdots \geq n_{d} \geq 2$. Let $\mathcal{S}=\operatorname{Seg}\left(\mathbb{P C}^{n_{1}} \times \mathbb{P C}^{n_{2}} \times \cdots \times \mathbb{P} \mathbb{C}^{n_{d}}\right)$ be the Segre variety in $\mathbb{P}\left(\mathbb{C}^{n_{1}} \otimes \mathbb{C}^{n_{2}} \otimes \cdots \otimes \mathbb{C}^{n_{d}}\right)$, and assume that $\bar{r}_{\mathcal{S}}$ is an integer. Then, $\mathcal{S}$ is not generically $\bar{r}_{\mathcal{S}}$-identifiable, unless it is one of the following cases:

1. $\mathcal{S}=\operatorname{Seg}\left(\mathbb{P C}^{5} \times \mathbb{P C}^{4} \times \mathbb{P C}^{3}\right)$, or

2. $\mathcal{S}=\operatorname{Seg}\left(\mathbb{P C}^{3} \times \mathbb{P} \mathbb{C}^{2} \times \mathbb{P} \mathbb{C}^{2} \times \mathbb{P} \mathbb{C}^{2}\right)$.

4. An effective criterion for specific identifiability. We formalize the concept of an effective criterion for specific identifiability.

Definition 9. Let $\mathcal{X} \subset \mathbb{P V}$ be a generically $r$-identifiable variety. A criterion for specific r-identifiability of $\mathcal{X}$ is called effective if it certifies identifiability on a dense subset of $\sigma_{r}(\mathcal{X})$ in the Euclidean topology.

Thus, if we consider a probability distribution with noncompact support on the affine cone of a generically $r$-identifiable variety $\mathcal{X}$, then the probability that an effective criterion for specific $r$-identifiability fails to certify identifiability of $p=p_{1}+\cdots+p_{r}$ is zero when the $p_{i}$ 's were randomly sampled from the probability distribution on $\widehat{\mathcal{X}}$.

4.1. The reshaped Kruskal criterion. We show that Kruskal's criterion [38] is effective when combined with reshaping. The key to this criterion is the notion of general linear position (GLP) [40]. A set of points $S=\left\{p_{1}, p_{2}, \ldots, p_{r}\right\} \subset \mathbb{P} V$ is in GLP if for $s=\min \{r, \operatorname{dim} V\}$, the subspace spanned by every subset $R \subset S$ of cardinality $s$ is of the maximal dimension $s-1$. This means that no 2 points coincide,

\footnotetext{
${ }^{4}$ This statement is true with probability 1 due to [33, Proposition 4.1] and [34, Section 5.1].
} 
no 3 points are on a line, no 4 points are on a plane, and so forth. The Kruskal rank of a finite set of points $S \subset \mathbb{P} V$ is then the largest value $\kappa$ for which every subset of $\kappa$ points of $S$ is in GLP.

Let $p_{i}=\mathbf{a}_{i}^{1} \otimes \cdots \otimes \mathbf{a}_{i}^{d}, i=1, \ldots, r$, be a collection of $r$ points in $\widehat{\mathcal{S}}$. Then we denote the factor matrices of the points $p_{i}$ by

$$
A_{k}=\left[\begin{array}{llll}
\mathbf{a}_{1}^{k} & \mathbf{a}_{2}^{k} & \cdots & \mathbf{a}_{r}^{k}
\end{array}\right]=\left[\begin{array}{llll}
\pi_{\{k\}}\left(p_{1}\right) & \pi_{\{k\}}\left(p_{2}\right) & \cdots & \pi_{\{k\}}\left(p_{r}\right)
\end{array}\right] \in \mathbb{F}^{n_{k} \times r}
$$

for $k=1,2, \ldots, d$. Letting $\mathbf{h} \subset\{1,2, \ldots, d\}$ be an ordered set, we define for brevity

$$
A_{\mathbf{h}}=A_{h_{1}} \odot A_{h_{2}} \odot \cdots \odot A_{h_{|\mathbf{h}|}}=\left[\begin{array}{llll}
\pi_{\mathbf{h}}\left(p_{1}\right) & \pi_{\mathbf{h}}\left(p_{2}\right) & \cdots & \pi_{\mathbf{h}}\left(p_{r}\right)
\end{array}\right] .
$$

Kruskal's criterion for specific identifiability may then be formulated as follows.

Proposition 10 (Kruskal's criterion [38]). Let $\mathcal{S}=\operatorname{Seg}\left(\mathbb{P F}^{n_{1}} \times \mathbb{P F}^{n_{2}} \times \mathbb{P F}^{n_{3}}\right)$ with $n_{1} \geq n_{2} \geq n_{3} \geq 2$. Let $p \in\left\langle p_{1}, p_{2}, \ldots, p_{r}\right\rangle$ with $p_{i}=\mathbf{a}_{i}^{1} \otimes \cdots \otimes \mathbf{a}_{i}^{d} \in \widehat{\mathcal{S}}$. Let $\kappa_{1}, \kappa_{2}$, and $\kappa_{3}$ denote the Kruskal ranks of the factor matrices $A_{1}, A_{2}$ and $A_{3}$ respectively. Then, $p$ is $r$-identifiable if $r \leq \frac{1}{2}\left(\kappa_{1}+\kappa_{2}+\kappa_{3}\right)-1$. Furthermore, this criterion is effective if $r \leq \frac{1}{2}\left(\min \left\{n_{1}, r\right\}+\min \left\{n_{2}, r\right\}+\min \left\{n_{3}, r\right\}\right)-1$, or, equivalently, letting $\delta=n_{2}+n_{3}-n_{1}-2$,

$$
r \leq n_{1}+\min \left\{\frac{1}{2} \delta, \delta\right\}
$$

this is the maximum range of applicability of Kruskal's criterion.

Proof. Effectiveness was not considered in [38], but its proof is a consequence of Lemma 12 that will be presented shortly.

Remark 11. While effectiveness of Kruskal's criterion is known to the experts, it is not obvious why this should have been expected. The reason is that Kruskal's criterion is not merely certifying the uniqueness of one decomposition

$$
p=p_{1}+\cdots+p_{r}=\sum_{i=1}^{r} \mathbf{a}_{i}^{1} \otimes \cdots \otimes \mathbf{a}_{i}^{d},
$$

with $p_{i} \in \widehat{\mathcal{S}}_{0}$ and $\mathbf{a}_{i}^{k} \in \mathbb{F}^{n_{k}}$, but rather it is testing whether all tensors $p=\alpha_{1} p_{1}+$ $\alpha_{2} p_{2}+\cdots+\alpha_{r} p_{r}, \alpha_{i} \in \mathbb{F}_{0}$ are $r$-identifiable. Indeed, the Kruskal rank of a set of points is a projective property: the Kruskal ranks of $\left\{\left[p_{1}\right], \ldots,\left[p_{r}\right]\right\}$ and $\left\{p_{1}, \ldots, p_{r}\right\}$ with $\left[p_{i}\right] \in \mathcal{S}$ are the same. This also means that Kruskal's test fails as soon as there exists one point $q=\alpha_{1} p_{1}+\alpha_{2} p_{2}+\cdots+\alpha_{r} p_{r}, \alpha_{i} \in \mathbb{F}_{0}$, that is not identifiable. Since all points $q=\alpha_{1} p_{1}+\alpha_{2} p_{2}+\cdots+\alpha_{r} p_{r}$ with some $\alpha_{i}=0$ are of rank at most $r-1$ and thus not $r$-identifiable, one could say that the $r$-secant plane $\left\langle\left[p_{1}\right],\left[p_{2}\right], \ldots,\left[p_{r}\right]\right\rangle, p_{i} \in \widehat{\mathcal{S}}_{0}$, is $r$-identifiable if and only if all elements of $\left\{\alpha_{1} p_{1}+\alpha_{2} p_{2}+\cdots+\alpha_{r} p_{r} \mid \alpha_{i} \in \mathbb{F}_{0}\right\}$ are $r$-identifiable. Kruskal's criterion is thus a criterion for checking that the $r$-secant plane $\left\langle\left[p_{1}\right],\left[p_{2}\right], \ldots,\left[p_{r}\right]\right\rangle$ is $r$-identifiable, when a particular tensor rank decomposition $p=p_{1}+p_{2}+\cdots+p_{r},\left[p_{i}\right] \in \mathcal{S}$, is provided as input.

We are not aware of criteria for specific $r$-identifiability that take into account the coefficients of the given decomposition. It is not inconceivable that for some high rank $r$, the secant space $\left\langle p_{1}, \ldots, p_{r}\right\rangle$ contains both $r$-identifiable and $r$-nonidentifiable points. Perhaps taking the coefficients into account could lead to criteria for specific identifiability that apply for higher ranks.

Consider a $d$-factor Segre product $\mathcal{S}=\operatorname{Seg}\left(\mathbb{F}^{n_{1}} \times \cdots \times \mathbb{F}^{n_{d}}\right)$ and let $\mathbf{h} \sqcup \mathbf{k} \sqcup \mathbf{l}=$ $\{1,2, \ldots, d\}$. Then, $\mathcal{S}=\operatorname{Seg}\left(\mathcal{S}_{\mathbf{h}} \times \mathcal{S}_{\mathbf{k}} \times \mathcal{S}_{\mathbf{l}}\right) \hookrightarrow \mathcal{S}_{\mathbf{h}, \mathbf{k}, \mathbf{l}}$, so an order-d rank-1 tensor of $\mathcal{S}$ can be viewed as an order-3 rank-1 tensor in $\mathcal{S}_{\mathbf{h}, \mathbf{k}, \mathbf{l}}$. We could try to apply Kruskal's 
criterion by interpreting $p \in \sigma_{r}^{0}(\mathcal{S})$ as a third-order tensor $p \in \sigma_{r}^{0}\left(\mathcal{S}_{\mathbf{h}, \mathbf{k}, \mathbf{l}}\right)$. Note that $\sigma_{r}(\mathcal{S})$ is a Zariski-closed subset ${ }^{5}$ of $\sigma_{r}\left(\mathcal{S}_{\mathbf{h}, \mathbf{k}, \mathbf{l}}\right)$ so that we cannot immediately conclude from Proposition 10 that Kruskal's criterion applied to reshaped tensors is effective. The range of effectiveness follows from the following result.

Lemma 12. Let $\mathcal{S}=\operatorname{Seg}\left(\mathbb{P F}^{n_{1}} \times \cdots \times \mathbb{P F}^{n_{d}}\right)$ with $\mathbb{F}=\mathbb{R}$ or $\mathbb{C}$. Then, there exists a Euclidean-dense, Zariski-open subset $G \subset \mathcal{S}^{\times r}$ such that for every nonempty $\mathbf{h} \subset$ $\{1,2, \ldots, d\}$ and every $\left(p_{1}, p_{2}, \ldots, p_{r}\right) \in G$, the points $\left(\pi_{\mathbf{h}}\left(p_{1}\right), \pi_{\mathbf{h}}\left(p_{2}\right), \ldots, \pi_{\mathbf{h}}\left(p_{r}\right)\right) \in$ $\mathcal{S}_{\mathbf{h}}$ are in $G L P$.

Proof. For $r=1$ the statement is obvious. So assume that $r \geq 2$.

We prove the existence of $G=G_{\{1,2, \ldots, d\}}$ by induction on the cardinality of $\mathbf{h}$. Specifically, we show that for every $\mathbf{h} \subset\{1,2, \ldots, d\}$ the configurations $\left(p_{1}, \ldots, p_{r}\right) \in$ $\mathcal{S}_{\mathbf{h}}$ that are not in GLP form a Zariski-closed subset $G_{\mathbf{h}} \subset \mathcal{S}_{\mathbf{h}}^{\times r}$. Let $\mathbf{h}=\{i\}$. Then, $\mathcal{S}_{\mathbf{h}}=\mathbb{P F}^{n_{i}}$. Let $s=\min \left\{n_{i}, r\right\}$. By definition, the configurations in $\mathcal{S}_{\mathbf{h}}^{\times r}$ wherein the first set of $s$ points are not in GLP can be described as

$$
\bigcup_{\left[q_{2}\right], \ldots,\left[q_{r}\right] \in \mathcal{S}_{\mathbf{h}}} \bigcup_{\alpha_{2}, \ldots, \alpha_{s} \in \mathbb{F}}\left(\left[\alpha_{2} q_{2}+\cdots+\alpha_{s} q_{s}\right],\left[q_{2}\right], \ldots,\left[q_{r}\right]\right) \subset \mathcal{S}_{\mathbf{h}}^{\times r},
$$

which can be obtained from a projection of $\mathbb{P F}^{s-1} \times \mathcal{S}_{\mathbf{h}}^{\times r-1}$, so that its dimension is strictly less than $\operatorname{dim} \mathcal{S}_{\mathbf{h}}^{\times r}$ because $\min \left\{r, n_{i}\right\}-2=\operatorname{dim} \mathbb{P F}^{s-1}<\operatorname{dim} \mathcal{S}_{\mathbf{h}}=n_{i}-1$. Hence (5) is a Zariski-closed set in $\mathcal{S}_{\mathbf{h}}^{\times r}$. The configurations in $\mathcal{S}_{\mathbf{h}}^{\times r}$ where $q_{i} \in$ $\mathcal{S}_{\mathbf{h}}$ is a linear combination of $s-1$ other points in $\mathcal{S}_{\mathbf{h}}$ can all be obtained from permuting the factors in the Cartesian product in (5). It follows that the union of all these Zariski-closed sets is precisely the Zariski-closed subset $G_{\mathbf{h}} \subset \mathcal{S}_{\mathbf{h}}^{\times r}$ of configurations $\left(q_{1}, \ldots, q_{r}\right) \in \mathcal{S}_{\mathbf{h}}^{\times r}$ that are not in GLP. Note that the sets $G_{\mathbf{h}}$ are $\mathbb{F}$-varieties because linear dependence of vectors can be formulated as a collection of determinantal equations with coefficients in $\mathbb{Z} \subset \mathbb{F}$.

Assume now that the statement is true for all $\mathbf{j} \subset\{1,2, \ldots, d\}$ whose cardinality is less than or equal to $k-1$. Then, we prove that it is true for every $\mathbf{h} \subset\{1,2, \ldots, d\}$ of cardinality $k$. Let $s=\min \left\{\prod_{i \in \mathbf{h}} n_{i}, r\right\}$. By induction, the sets $G_{\mathbf{j}}$ with $\mathbf{j} \subsetneq \mathbf{h}$ are Zariski-closed. Consider the surjective map

$$
\begin{aligned}
\left(\mathcal{S}_{\mathbf{j}}^{\times r} \backslash G_{\mathbf{j}}\right) \times\left(\mathcal{S}_{\mathbf{h} \backslash \mathbf{j}}^{\times r} \backslash G_{\mathbf{h} \backslash \mathbf{j}}\right) & \rightarrow \mathcal{S}_{\mathbf{h}}^{\times r} \backslash H_{\mathbf{h}, \mathbf{j}} \\
\left(\left[x_{1}\right],\left[x_{2}\right], \ldots,\left[x_{r}\right]\right) \times\left(\left[y_{1}\right],\left[y_{2}\right], \ldots,\left[y_{r}\right]\right) & \mapsto\left(\left[x_{1} \otimes y_{1}\right],\left[x_{2} \otimes y_{2}\right], \ldots,\left[x_{r} \otimes y_{r}\right]\right),
\end{aligned}
$$

where $H_{\mathbf{h}, \mathbf{j}}$ can be defined as

$$
H_{\mathbf{h}, \mathbf{j}}=\left\{\left(\left[x_{1} \otimes y_{1}\right], \ldots,\left[x_{r} \otimes y_{r}\right]\right) \mid\left(\left[x_{1}\right], \ldots,\left[x_{r}\right]\right) \in G_{\mathbf{j}} \text { or }\left(\left[y_{1}\right], \ldots,\left[y_{r}\right]\right) \in G_{\mathbf{h} \backslash \mathbf{j}}\right\} .
$$

Let $\Pi_{\mathbf{l}}=\prod_{i \in \mathbf{l}} n_{i}$ for any $\mathbf{l} \subset\{1,2, \ldots, d\}$. Let a Gröbner basis of the ideal of $G_{\mathbf{j}}$ consist of the $\mathbb{F}$-polynomials $f_{i}\left(x_{1,1}, x_{2,1}, \ldots, x_{\Pi_{\mathbf{j}}, 1}, \ldots, x_{1, r}, x_{2, r}, \ldots, x_{\Pi_{\mathbf{j}}, r}\right)$, and similarly let $g_{i}\left(y_{1,1}, y_{2,1}, \ldots, y_{\Pi_{\mathbf{h} \backslash \mathbf{j}}, 1}, \ldots, y_{1, r}, y_{2, r}, \ldots, y_{\Pi_{\mathbf{h} \backslash \mathbf{j}}, r}\right)$ be the polynomials in a Gröbner basis of the ideal of $G_{\mathbf{h} \backslash \mathbf{j}}$. Let $Z_{i, j, k}=x_{i, k} y_{j, k}$ with $i=1, \ldots, \Pi_{\mathbf{j}}, j=$ $1, \ldots, \Pi_{\mathbf{h} \backslash \mathbf{j}}$, and $k=1, \ldots, r$ be variables for $\mathcal{S}_{\mathbf{h}}^{\times r}$. Then, $H_{\mathbf{h}, \mathbf{j}} \subset \mathcal{S}_{\mathbf{h}}^{\times r}$ is contained in the variety whose ideal is spanned by the following set of $\mathbb{F}$-polynomials:

$$
\begin{aligned}
f_{i}\left(Z_{1, \mu, 1}, \ldots, Z_{\Pi_{\mathbf{j}}, \mu, 1}, \ldots, Z_{1, \mu, r}, \ldots, Z_{\Pi_{\mathbf{j}}, \mu, r}\right) & \\
& g_{j}\left(Z_{\nu, 1,1}, \ldots, Z_{\nu, \Pi_{\mathbf{h} \backslash \mathbf{j}}, 1}, \ldots, Z_{\nu, 1, r}, \ldots, Z_{\nu, \Pi_{\mathbf{h} \backslash \mathbf{j}}, r}\right)
\end{aligned}
$$

\footnotetext{
${ }^{5}$ We are assuming here that $\mathcal{S}_{\mathbf{h}, \mathbf{k}, \mathbf{l}}$ is nondefective [1].
} 
for every $(i, j), \mu=1,2, \ldots, \Pi_{\mathbf{h} \backslash \mathbf{j}}$, and $\nu=1,2, \ldots, \Pi_{\mathbf{j}}$. As $G_{\mathbf{j}}$ is Zariski-closed by induction, $H_{\mathbf{h}, \mathbf{j}}$ is Zariski-closed. Thus the finite union $H_{\mathbf{h}}=\bigcup_{\mathbf{j} \subset \mathbf{h}} H_{\mathbf{h}, \mathbf{j}}$ is a Zariskiclosed set. Now, $\mathcal{S}_{\mathbf{h}}^{\times r} \backslash H_{\mathbf{h}}$ contains all configurations $\left(p_{1}, p_{2}, \ldots, p_{r}\right)$ for which for every $\mathbf{j} \subsetneq \mathbf{h}$ we have that $\left(\pi_{\mathbf{j}}\left(p_{1}\right), \pi_{\mathbf{j}}\left(p_{2}\right), \ldots, \pi_{\mathbf{j}}\left(p_{r}\right)\right)$ is in GLP. As in the proof of the base case, it is straightforward to show that there exists a Zariski-closed set $G_{\mathbf{h}}^{\prime} \subset \mathcal{S}_{\mathbf{h}}^{\times r}$ that contains all configurations that are not in GLP. The proof is then concluded by setting $G_{\mathbf{h}}=G_{\mathbf{h}}^{\prime} \cup H_{\mathbf{h}}$.

The foregoing result has some implications for the Khatri-Rao product that could be of independent interest, generalizing [37, Corollary 1] to the real case.

Corollary 13. Let $\left(A_{1}, A_{2}, \ldots, A_{d}\right) \in \mathbb{F}^{n_{1} \times r} \times \mathbb{F}^{n_{2} \times r} \times \cdots \times \mathbb{F}^{n_{d} \times r}$ be generic. Then, for every $\mathbf{h} \subset\{1,2, \ldots, d\}$ of cardinality $k>0$ the matrix $A_{h_{1}} \odot A_{h_{2}} \odot \cdots \odot A_{h_{k}}$ has the maximal rank, i.e., $\min \left\{r, \prod_{i \in \mathbf{h}} n_{i}\right\}$.

It follows immediately from Proposition 10 and Lemma 12 that Kruskal's theorem with reshaping is effective in the broadest range that one could have expected.

TheOREm 14 (Reshaped Kruskal criterion). Let $d \geq 3$, and let $\mathcal{S}=\operatorname{Seg}\left(\mathbb{P F}^{n_{1}} \times\right.$ $\left.\mathbb{P F}^{n_{2}} \times \cdots \times \mathbb{P F}^{n_{d}}\right)$, and let $p \in\left\langle p_{1}, p_{2}, \ldots, p_{r}\right\rangle$ with $p_{i}=\mathbf{a}_{i}^{1} \otimes \cdots \otimes \mathbf{a}_{i}^{d} \in \widehat{\mathcal{S}}$. Let $\Pi_{\mathbf{m}}=\prod_{\ell \in \mathbf{m}} n_{\ell}$ for any $\mathbf{m} \subset\{1,2, \ldots, d\}$. Let $\mathbf{h} \sqcup \mathbf{k} \sqcup \mathbf{l}=\{1,2, \ldots, d\}$ be such that $\Pi_{\mathbf{h}} \geq \Pi_{\mathbf{k}} \geq \Pi_{\mathbf{l}}$. Let the Kruskal ranks of the factor matrices $A_{\mathbf{h}}, A_{\mathbf{k}}$ and $A_{\mathbf{l}}$ be denoted by $\kappa_{1}, \kappa_{2}$ and $\kappa_{3}$ respectively. Then, $p$ is r-identifiable if $r \leq \frac{1}{2}\left(\kappa_{1}+\kappa_{2}+\kappa_{3}\right)-1$. Furthermore, letting $\delta=\Pi_{\mathbf{k}}+\Pi_{\mathbf{l}}-\Pi_{\mathbf{h}}-2$, this criterion is effective if

$$
r \leq \Pi_{\mathbf{h}}+\min \left\{\frac{1}{2} \delta, \delta\right\} .
$$

4.2. A heuristic for reshaping. Choosing the partition $\mathbf{h} \sqcup \mathbf{k} \sqcup \mathbf{l}$ in Theorem 14 influences the range in which the criterion is effective. Note that if $\Pi_{\mathbf{h}} \geq r \geq \Pi_{\mathbf{k}} \geq \Pi_{\mathbf{l}}$, then the criterion in Theorem 14 is effective for $r \leq \Pi_{\mathbf{k}}+\Pi_{\mathbf{l}}-2$. After our discussions with I. Domanov, we realized that a good heuristic yielding a large effective range of identifiability consists of first choosing

$$
\mathbf{k} \in \underset{\substack{\mathbf{y} \subset\{1, \ldots, d\}, \mathbf{x} \sqcup \mathbf{y} \sqcup \mathbf{z}=\{1, \ldots, d\}, \Pi_{\mathbf{x}} \geq \Pi_{\mathbf{y}} \geq \Pi_{\mathbf{z}}}}{\arg \max } \Pi_{\mathbf{y}}, \quad \text { and then } \mathbf{h} \in \underset{\substack{\mathbf{x} \subset\{1, \ldots, d\}, \mathbf{x} \cup \mathbf{k} \sqcup \mathbf{z}=\{1, \ldots, d\}, \Pi_{\mathbf{x}} \geq \Pi_{\mathbf{k}} \geq \Pi_{\mathbf{z}}}}{\arg \min } \Pi_{\mathbf{x}},
$$

and finally $\mathbf{l}=\{1,2, \ldots, d\} \backslash(\mathbf{h} \cup \mathbf{k})$. One should thus first try to maximize the secondlargest reshaped dimension $\Pi_{\mathbf{k}}$, and then minimize the largest reshaped dimension.

Example 15. Let $d=4$. Then there are 6 distinct partitions of $\{1,2,3,4\}$, namely $\sigma_{1,2}=\{1,2\} \sqcup\{3\} \sqcup\{4\}, \sigma_{1,3}=\{1,3\} \sqcup\{2\} \sqcup\{4\}, \sigma_{1,4}=\{1,4\} \sqcup\{2\} \sqcup\{3\}, \sigma_{2,3}=$ $\{2,3\} \sqcup\{1\} \sqcup\{4\}, \sigma_{2,4}=\{2,4\} \sqcup\{1\} \sqcup\{3\}$, and $\sigma_{3,4}=\{3,4\} \sqcup\{1\} \sqcup\{2\}$. The effective range of the reshaped Kruskal criterion in Theorem 14 corresponding to these partitions is given below for a few arbitrarily chosen shapes:

\begin{tabular}{ccccccc}
\hline$\left(n_{1}, n_{2}, n_{3}, n_{4}\right)$ & $\sigma_{1,2}$ & $\sigma_{1,3}$ & $\sigma_{2,3}$ & $\sigma_{1,4}$ & $\sigma_{2,4}$ & $\sigma_{3,4}$ \\
\hline$(17,13,13,2)$ & 13 & 13 & 17 & 24 & $\mathbf{2 7}$ & $\mathbf{2 7}$ \\
$(17,8,3,2)$ & 3 & 8 & $\mathbf{1 7}$ & 9 & 17 & 12 \\
$(15,15,11,10)$ & 19 & 23 & 23 & 24 & 24 & $\mathbf{2 8}$ \\
$(15,13,9,4)$ & 11 & 15 & 17 & 20 & 22 & $\mathbf{2 6}$ \\
$(12,10,7,7)$ & 12 & 15 & 17 & 15 & 17 & $\mathbf{2 0}$ \\
\hline
\end{tabular}

The values highlighted in bold correspond to the choice of the heuristic. In all of these examples, the heuristic choice resulted in the largest range for which the reshaped Kruskal criterion could be applied. 
The heuristic is asymptotically optimal in two extreme cases, namely when $\mathcal{S}$ is unbalanced and when $n_{1}=n_{2}=\cdots=n_{d}=n$. We expect that the proposed partitioning should perform reasonably well in other instances as well.

Proposition 16. Let $\mathcal{S}=\operatorname{Seg}\left(\mathbb{P F}^{n} \times \cdots \times \mathbb{P F}^{n}\right)$ be a d-factor Segre product. Then the reshaped Kruskal criterion is effective for

$$
r \leq \begin{cases}\frac{3}{2} n-1 & \text { if } d=3, \\ 2 n-2 & \text { if } d=4, \\ n^{\lfloor(d-1) / 2\rfloor}+\frac{1}{2} n^{d-2\lfloor(d-1) / 2\rfloor}-1 & \text { if } d \geq 5 .\end{cases}
$$

Furthermore, for large $n$ this is the largest range in which Theorem 14 applies.

Proof. The case $d=3$ is Proposition 10.

In the case $d=4$, the only admissible reshaping, up to a permutation of the factors, is to a $n^{2} \times n \times n$ tensor. An application of Theorem 14 yields the result. Since it is the only admissible reshaping, it is optimal.

Let $d \geq 5$. Let the cardinality of $\mathbf{h}, \mathbf{k}$, and $\mathbf{l}$ be respectively $\alpha, \beta$, and $\gamma$, where $\alpha+\beta+\gamma=d$ and $\alpha \geq \beta \geq \gamma \geq 1$. Suppose first that $r \geq n^{\alpha} \geq n^{\beta} \geq n^{\gamma}$, so that the criterion is effective if $n^{\alpha} \leq r \leq \frac{1}{2}\left(n^{\alpha}+n^{\beta}+n^{\gamma}\right)-1$. For sufficiently large $n$, these inequalities are consistent only if $\alpha=\beta \geq \gamma$. In this case, the criterion would be effective up to $r \leq n^{\alpha}+\frac{1}{2} n^{\gamma}-1$. If $n$ is sufficiently large, the optimal case is obtained when $\alpha=\beta=\lfloor(d-1) / 2\rfloor$ and $\gamma=d-2 \alpha$. This is precisely what one obtains by applying the proposed heuristic. Indeed, in the first step we would choose $\alpha \geq \beta=\lfloor(d-1) / 2\rfloor$. Then, $\alpha$ could either be $\lfloor(d-1) / 2\rfloor$ or $\lceil(d-1) / 2\rceil$ with the heuristic suggesting to pick $\alpha=\beta$. Finally, the value of $\gamma$ is set to $d-2 \alpha$ so that $\gamma \leq 2 \leq \beta \leq \alpha$. The remaining configurations do not result in a larger range of effective identifiability. If $n^{\alpha} \geq r \geq n^{\beta} \geq n^{\gamma}$, then the reshaped Kruskal criterion is effective for $r \leq n^{\beta}+n^{\gamma}-2$. There is but one choice of $\beta$ that might result in a larger range than the proposed heuristic, namely $\beta=\lfloor(d-1) / 2\rfloor, \alpha=\lceil(d-1) / 2\rceil$ and $\gamma=1$, and this can only occur when $d$ is even. However, the resulting range is not optimal because $n \leq \frac{1}{2} n^{d-2\lfloor(d-1) / 2\rfloor}=\frac{1}{2} n^{2}$ (whenever $n \geq 2$ ) for even $d$, so that the proposed heuristic always covers a wider range. If $n^{\alpha} \geq n^{\beta} \geq r$, then the criterion is effective for $r \leq n^{\beta}$, but it is immediately clear that this range is not optimal.

Proposition 17. Let $\mathcal{S}=\operatorname{Seg}\left(\mathbb{P F}^{n_{1}} \times \cdots \times \mathbb{P F}^{n_{d}}\right)$ with $n_{1} \geq \cdots \geq n_{d} \geq 3$ be an unbalanced Segre variety, i.e., $n_{1}>1+\prod_{i=2}^{d} n_{d}-\sum_{i=2}^{d}\left(n_{i}-1\right)$. Then the reshaped Kruskal criterion in Theorem 14 is effective for

$$
r \leq \prod_{i=2}^{d-1} n_{i}+n_{d}-2 .
$$

Furthermore, this is the largest range in which Theorem 14 applies.

Proof. For $d=3$, we may apply Proposition 10. Since the case $r \geq n_{1}$ is not generically $r$-identifiable because of [18, Theorem 3.1] and [15, Proposition 8.2], it follows that $r \leq \frac{1}{2}\left(r+n_{2}+n_{3}\right)-1$ is the widest range in which Kruskal's criterion applies, concluding the proof in this case.

Let $d \geq 4$ in the remainder. Assume that $n_{1}<\prod_{i=2}^{d-1} n_{i}$. Then, we observe that

$$
\prod_{i=2}^{d-1} n_{i}>n_{1}>1+\prod_{i=2}^{d} n_{i}-\sum_{i=2}^{d}\left(n_{i}-1\right)
$$


is inconsistent, as we should have that

$$
\begin{aligned}
1 & >n_{d}\left(1-\prod_{i=2}^{d-1} n_{i}^{-1}\right)-\frac{\sum_{i=2}^{d-1}\left(n_{i}-1\right)}{\prod_{i=2}^{d-1} n_{i}}+2 \prod_{i=2}^{d-1} n_{i}^{-1} \\
& =n_{d}\left(1-\prod_{i=2}^{d-1} n_{i}^{-1}\right)-\frac{\sum_{i=2}^{d-1} n_{i}}{\prod_{i=2}^{d-1} n_{i}}+d \prod_{i=2}^{d-1} n_{i}^{-1} \geq 3-(d-1) 3^{-d+3}
\end{aligned}
$$

where the second inequality is because of $n_{i} \geq 3$. However, the right hand side is never less than 1 if $d \geq 4$, so that $n_{1} \geq \prod_{i=2}^{d-1} n_{i}$. It follows that the heuristic chooses $\mathbf{h}=\{1\}, \mathbf{k}=\{2, \ldots, d-1\}$, and $\mathbf{l}=\{d\}$.

It follows from $n_{1} \geq \prod_{i=2}^{d-1} n_{i}$ that $n_{1}$ is larger than every $\Pi_{\mathbf{k}}$ with $\{1\} \sqcup \mathbf{k} \sqcup$ $\mathbf{l}=\{1, \ldots, d\}$ with both $\mathbf{k}$ and $\mathbf{l}$ nonempty. So, the conditions in Theorem 14 can be satisfied only if $\mathbf{h} \subset\{1, \ldots, d\}$ contains at least "1." Whatever the partition $\mathbf{h} \sqcup \mathbf{k} \sqcup \mathbf{l}=\{1, \ldots, d\}$ with $1 \in \mathbf{h}$, we must have $\delta<0$ because otherwise the criterion would be effective for $r$ larger than $n_{1}$. Therefore, the effective range of identifiability of Theorem 14 is $r \leq \Pi_{\mathbf{k}}+\Pi_{\mathbf{l}}-2$ with $\Pi_{\mathbf{k}} \geq \Pi_{\mathbf{l}}$ and where $\mathbf{k} \sqcup \mathbf{l}=\{1, \ldots, d\} \backslash \mathbf{h}$. It follows from $n_{1} \geq \cdots \geq n_{d} \geq 3$ and the observation that $n_{i} a+\frac{1}{n_{i}} b>a+b$ when $n_{i} \geq 3$ and $a \geq b$ that the maximum is reached for $\mathbf{k}=\{2, \ldots, d-1\}$, which is precisely the choice of the heuristic. This concludes the proof.

5. Symmetric identifiability. This section introduces a technique for investigating specific identifiability in the symmetric setting based on the Hilbert function.

5.1. Basic results. A well-known result on effective symmetric identifiability is the catalecticant method of $[35,5.4]$. It is stated below only for the even degree case as the reshaped Kruskal criterion applies in a wider range for odd degree.

Proposition 18 (Iarrobino and Kanev [35]). Let $d=2 m$, and let $\mathcal{V}=\mathbb{P} v_{d}\left(\mathbb{F}^{n+1}\right)$ be the Veronese variety. Let $p=p_{1}+\cdots+p_{r}$ with $p_{i}=\mathbf{a}_{i}^{\text {od }} \in \widehat{\mathcal{V}}$ be a given decomposition. Let the most square symmetric flattening of $p$ be denoted by

$$
C=\sum_{i=1}^{r}\left(\mathbf{a}_{i}^{\circ m}\right)\left(\mathbf{a}_{i}^{\circ m}\right)^{T} .
$$

If $\operatorname{rank}(C)=r$ and $r \leq\left(\begin{array}{c}n+m \\ m\end{array}\right)-(n+1)$, then the kernel of $C$ is the ideal $I_{Z, m}$ of polynomials of degree $m$ simultaneously vanishing on $Z=\left\{\mathbf{a}_{1}, \ldots, \mathbf{a}_{r}\right\}$. If additionally the degree of the closure of the zero set of $I_{Z, m}$ is $r$, then $p$ is r-identifiable. This criterion is effective for all

$$
r \leq\left(\begin{array}{c}
n+m \\
m
\end{array}\right)-(n+1)
$$

Proof. Effectiveness was proved in [45, Theorem 2.4].

An implementation of the catalecticant method - which is easily adapted to a criterion for effective specific identifiability as outlined above - is also described in [45].

The reshaped Kruskal criterion for general tensors is also effective when applied to reshaped symmetric tensors. If $d_{1}+d_{2}+d_{3}=d$ is a partition of $d$, then reshaping a rank-1 symmetric tensor can be thought of as

$$
\mathbb{P} v_{d}\left(\mathbb{F}^{n+1}\right) \rightarrow \operatorname{Seg}\left(\mathbb{P} v_{d_{1}}\left(\mathbb{F}^{n+1}\right) \times \mathbb{P} v_{d_{2}}\left(\mathbb{F}^{n+1}\right) \times \mathbb{P} v_{d_{3}}\left(\mathbb{F}^{n+1}\right)\right)
$$




$$
\left[\mathbf{a}_{i}^{\otimes d}\right] \mapsto\left[\mathbf{a}_{i}^{\otimes d_{1}} \otimes \mathbf{a}_{i}^{\otimes d_{2}} \otimes \mathbf{a}_{i}^{\otimes d_{3}}\right]
$$

The map can be extended linearly to define reshaping for an arbitrary $d$-form. The image of this map is contained in the projectivization of $S^{d_{1}} \mathbb{F}^{n+1} \otimes S^{d_{2}} \mathbb{F}^{n+1} \otimes S^{d_{3}} \mathbb{F}^{n+1} \cong$ $\mathbb{F}^{\left(\begin{array}{c}n+d_{1} \\ d_{1}\end{array}\right)} \otimes \mathbb{F}^{\left(\begin{array}{c}n+d_{2} \\ d_{2}\end{array}\right)} \otimes \mathbb{F}^{\left(\begin{array}{c}n+d_{3} \\ d_{3}\end{array}\right) .}$

Lemma 19. Let $\mathcal{S}=\operatorname{Seg}\left(\mathbb{P F}^{n+1} \times \cdots \times \mathbb{P F}^{n+1}\right)$ be a d-factor Segre variety. Let $\mathcal{V}=$ $\mathbb{P} S^{d} \mathbb{F}^{n+1} \cap \mathcal{S}$ be the variety of symmetric rank-1 tensors in $\mathbb{P}\left(\mathbb{F}^{n+1} \otimes \cdots \otimes \mathbb{F}^{n+1}\right)$. Then, there exists a dense, Zariski-open subset $G \subset \mathcal{V}^{\times r}$ with the property that for every $\mathbf{h} \subset$ $\{1,2, \ldots, d\}$ and every $\left(p_{1}, p_{2}, \ldots, p_{r}\right) \in G$, the points $\left(\pi_{\mathbf{h}}\left(p_{1}\right), \pi_{\mathbf{h}}\left(p_{2}\right), \ldots, \pi_{\mathbf{h}}\left(p_{r}\right)\right) \in$ $\mathcal{S}_{\mathbf{h}} \cap \mathbb{P} S^{|\mathbf{h}|} \mathbb{F}^{n+1}$ are in $G L P$.

Proof. The proof follows along the same lines as the proof of Lemma 12.

The foregoing lemma in combination with Proposition 10 yields a symmetric version of the reshaped Kruskal condition in Theorem 14.

Corollary 20. Let $\mathcal{S}=\operatorname{Seg}\left(\mathbb{P F}^{n+1} \times \cdots \times \mathbb{P F}^{n+1}\right)$ and $\mathcal{V}=\mathbb{P} S^{d} \mathbb{F}^{n+1} \cap \mathcal{S}$. Let $p \in\left\langle p_{1}, \ldots, p_{r}\right\rangle$ with $p_{i}=\mathbf{a}_{i}^{\otimes d} \in \widehat{\mathcal{V}}$. Let $\Gamma_{k}=\left(\begin{array}{c}k+n \\ n\end{array}\right)$ for $k \in \mathbb{N}$. Let $d_{1}+d_{2}+d_{3}=d$ be a partition of $d$, such that $d_{1} \geq d_{2} \geq d_{3}$. Let $\kappa_{1}, \kappa_{2}$, and $\kappa_{3}$ denote the Kruskal ranks of $\left\{\mathbf{a}_{1}^{\otimes d_{1}}, \ldots, \mathbf{a}_{r}^{\otimes d_{1}}\right\},\left\{\mathbf{a}_{1}^{\otimes d_{2}}, \ldots, \mathbf{a}_{r}^{\otimes d_{2}}\right\}$, and $\left\{\mathbf{a}_{1}^{\otimes d_{3}}, \ldots, \mathbf{a}_{r}^{\otimes d_{3}}\right\}$ respectively. Then, $p$ is $r$-identifiable if $r \leq \frac{1}{2}\left(\kappa_{1}+\kappa_{2}+\kappa_{3}\right)-1$. Furthermore, letting $\delta=\Gamma_{d_{2}}+\Gamma_{d_{3}}-\Gamma_{d_{1}}-2$, this criterion is effective if

$$
r \leq \Gamma_{d_{1}}+\min \left\{\frac{1}{2} \delta, \delta\right\}
$$

For large $n$, the maximum range of effective $r$-identifiability is attained for $d_{1}=d_{2}=$ $\left\lfloor\frac{1}{2}(d-1)\right\rfloor$ and $d_{3}=d-2 d_{1}$ :

$$
r \leq \begin{cases}\frac{3}{2} n+\frac{1}{2} & \text { if } d=3 \\
2 n & \text { if } d=4, \\
\left(\begin{array}{c}
d_{1}+n \\
d_{1}
\end{array}\right)+\frac{1}{2}\left(\begin{array}{c}
d_{3}+n \\
d_{3}
\end{array}\right)-1 & \text { if } d \geq 5\end{cases}
$$

Proof. The upper bound on the range of effective identifiability can be proved in exactly the same way as Proposition 16.

This criterion is completely effective for the spaces listed in Theorem 2.

Proof of Theorem 2, part I. $S^{3} \mathbb{F}^{3}$ is the only "normal" case in the theorem. It is generically $r$-identifiable for $r \leq 3$, the generic rank is 4 and the space is not perfect (or equiabundant). Corollary 20 applies up to 3 , hence concluding this case.

The space $S^{3} \mathbb{F}^{4}$ is perfect and one of the exceptionally identifiable cases in Theorem 5. Generic $r$-identifiability holds up to $r=5$ and Corollary 20 establishes effective specific identifiability up to $r=5$ as well.

$S^{3} \mathbb{F}^{5}$ is a perfect space with generic rank 7 that is not generically 7 -identifiable because of Theorem 5. It is generically $r$-identifiable for $r \leq 6$ and Corollary 20 is an effective criterion in this range.

Both $S^{3} \mathbb{F}^{6}$ and $S^{6} \mathbb{F}^{3}$ are effectively identifiable because Corollary 20 applies up to $r=8$, both $\mathbb{P} v_{3}\left(\mathbb{F}^{6}\right)$ and $\mathbb{P} v_{6}\left(\mathbb{F}^{3}\right)$ are generically $r$-identifiable for $r \leq 8$, and they are 9-tangentially weakly defective by Theorem 3 .

$S^{4} \mathbb{F}^{3}$ is a perfect space with generic rank 5 . Corollary 20 yields an effective specific identifiability criterion up to $r=4$. Since $S^{4} \mathbb{F}^{3}$ is not 5 -identifiable by Theorem 5 , the proof of this case is concluded. 
Binary forms of even degree $\mathbb{P} v_{2 k}\left(\mathbb{F}^{2}\right)$ are generically $k$-identifiable, but not identifiable for the generic rank $k+1$. For $k=2$, Corollary 20 yields effective specific identifiability up to 2 . For $k \geq 3$, taking $d_{1}=d_{2}=k-1$ and $d_{3}=2$, Corollary 20 implies effective specific identifiability up to $r \leq(1+k-1)+\frac{1}{2}(1+2)-1=k+\frac{1}{2}$. For $\mathbb{P} v_{2 k+1}\left(\mathbb{F}^{2}\right)$ generic $r$-identifiability exceptionally holds up to $r=k+1$ by Theorem 5 . Corollary 20 then implies effective specific $r$-identifiability up to $r \leq(k+1)+\frac{1}{2} 2-1=k+1$ by choosing $d_{1}=d_{2}=k$ and $d_{3}=1$ if $d \geq 5$; the case $d=3$ yields $r \leq 2$. This proves effective identifiability of $S^{d} \mathbb{F}^{2}$ for all $d \geq 3$.

From [45, Theorem 3.5(2)], it follows that $S^{3} \mathbb{F}^{5}$ is effectively $r$-identifiable via [45, Algorithm 3] up to rank $r=7$, which is the generic rank. It is interesting to note that the space $S^{3} \mathbb{F}^{5}$ is exceptionally perfect identifiable [29].

$S^{7} \mathbb{F}^{3}$ is a perfect space with generic rank 12 . It is not exceptionally generically 12-identifiable because of [29]. Algorithm 3 of [45] provides an effective criterion for $r$-identifiability up to $r=11$, by [45, Theorem 3.5(2)].

5.2. The Hilbert function. In this section we introduce some algebraic methods for the detection of the identifiability of symmetric tensors, namely the Hilbert function of a set of points in a projective space and their $h$-vector. Both of these methods are widely used in algebraic geometry, and their application to the identifiability problem has been considered before in the literature; see, e.g., [7, 8, 14, 17]. Yet, we believe that the interactions between the Hilbert function and tensor analysis have not yet been fully explored (see also [19]). We will employ these techniques in the next section for proving the last remaining case of Theorem 2.

Consider a polynomial ring $R=\mathbb{C}\left[x_{0}, \ldots, x_{n}\right]$ and the linear space $R_{d}$ of forms of degree $d$. Let $Z$ be a finite set in $\mathbb{P} \mathbb{C}^{n+1}$. Call $I_{Z}$ the homogeneous ideal of the set $Z$. Then there is an exact sequence of graded modules: $0 \rightarrow I_{Z} \rightarrow R \rightarrow R / I_{Z} \rightarrow 0$.

Definition 21. The Hilbert function $H_{Z}$ of the set $Z$ associates to each integer $d$ the dimension $H_{Z}(d)$ of the linear space $\left(R / I_{Z}\right)_{d}$.

Remark 22. There is an interpretation of the Hilbert function in terms of the residue of forms at points. For a form $f \in R_{d}$ and a point $P \in Z$, the evaluation $f(P)$ is not well defined, as it depends on the choice of coordinates for $P$, which is fixed only up to scalar multiplication. However, if we consider the residues of all forms in a linear space at all possible homogeneous coordinates of the points of $Z$, then we get a well defined subspace of $\mathbb{C}^{\ell}$, where $\ell$ is the cardinality of $Z$. In this sense, if we take the residue of all forms of degree $d$, the dimension of the subspace of $\mathbb{C}^{\ell}$ that we obtain is equal to $H_{Z}(d)$.

A precise algebraic formulation of this principle is easy in the theory of sheaves. Call $\mathcal{O}$ the structure sheaf of $\mathbb{P} \mathbb{C}^{n+1}$ and $\mathcal{O}_{Z}$ the structure sheaf of $Z$, which is a skyscraper sheaf supported at the $\ell$ points of $Z$. Then for any degree $d$ we have a well-defined surjective map of sheaves $\mathcal{O}(d) \rightarrow \mathcal{O}_{Z}$ whose kernel is the ideal sheaf $\mathcal{I}_{Z}(d)$ of $Z$. Taking global sections, we get an exact sequence of vector spaces $0 \rightarrow$ $H^{0}(\mathcal{I}) \rightarrow H^{0}(\mathcal{O}(d)) \rightarrow H^{0}\left(\mathcal{O}_{Z}\right)$. Since $\mathcal{O}_{Z}$ is a skyscraper sheaf, then $H^{0}\left(\mathcal{O}_{Z}\right)$ can be non-canonically identified with $\mathbb{C}^{\ell}$, while $H^{0}(\mathcal{O}(d))$ is $R_{d}$. The left-hand map $\rho_{d}: H^{0}(\mathcal{O}(d)) \rightarrow H^{0}\left(\mathcal{O}_{Z}\right)$ corresponds to taking residues, as specified above. Thus the rank of $\rho_{d}$ is the value of the Hilbert function $H_{Z}(d)$.

Some elementary properties of the Hilbert function are recalled next; see, e.g., [52] or [31, Section 2].

Proposition 23.

(i) $0=H_{Z}(-1)=H_{Z}(-2)=\ldots$; 
(ii) $H_{Z}(0)=1$;

(iii) $H_{Z}(1)<n+1$ exactly when $Z$ is contained in a hyperplane;

(iv) $H_{Z}(d)<\left(\begin{array}{c}n+d \\ d\end{array}\right)$ if and only if $Z$ is contained in a hypersurface of degree $d$;

(v) $H_{Z}(d) \leq H_{Z}(d+1)$;

(vi) $H_{Z}(d)$ cannot be bigger than the cardinality $\ell$ of $Z$;

(vii) for all $d \gg 0$ then $H_{Z}(d)=\ell$, the cardinality of $Z$; and

(viii) if $Z^{\prime} \subset Z$ then $H_{Z}(d) \geq H_{Z^{\prime}}(d)$ for all $d$.

From now on, we write $\ell_{Z}$ for the cardinality of a finite set $Z$. A bit more difficult, but still straightforward, is the proof of the next property, which is a consequence of Theorem 3.6 in [12] where a much more precise result is proved.

Proposition 24. If $H_{Z}\left(d_{0}\right)=H_{Z}\left(d_{0}+1\right)$ for some $d_{0} \geq 0$, then $H_{Z}(d)=\ell_{Z}$ for all $d \geq d_{0}$.

By Proposition $23(\mathrm{v}), h_{Z}(d)=H_{Z}(d)-H_{Z}(d-1)$ is always non-negative. Furthermore, by (i) and (ii) of Proposition 23 we get $h_{Z}(0)=1$, and from Proposition 24 it follows that if $h_{Z}(d)=0$ for some $d>0$, then $h_{Z}\left(d^{\prime}\right)=0$ for all $d^{\prime} \geq d$.

Definition 25. Let $Z$ be a finite set. The $h$-vector of $Z$ is the sequence of integers $\left(h_{Z}(0), h_{Z}(1), \ldots, h_{Z}(c)\right)$ where $c$ is the maximum such that $h_{Z}(c)>0$.

The basic properties of the $h$-vector, which are consequences of Propositions 23 and 24 , can be summarized as follows.

Proposition 26.

(i) $h_{Z}(0)=1$;

(ii) $h_{Z}(i)>0$ for all $i$;

(iii) $h_{Z}(1)$ is the dimension of the projective linear span of $Z$;

(iv) If $\left(h_{Z}(0), \ldots, h_{Z}(c)\right)$ is the $h$-vector of $Z$, then $H_{Z}(c)=\ell_{Z}$ and $H_{Z}(i)<\ell_{Z}$ for $i=0, \ldots, c-1$; and

(v) $\sum_{i=0}^{c} h_{Z}(i)=H_{Z}(c)=\ell_{Z}$.

Proposition 27. If $Z^{\prime} \subset Z$ then $h_{Z^{\prime}}(d) \leq h_{Z}(d)$ for all $d$.

Proof. The $h$-vector $h_{Z}$ of $Z$ corresponds to the Hilbert function of an Artinian reduction $R /\left(\mathcal{I}_{Z}+L\right)$ with $L$ a generic linear form (see e.g. [43, Remark 6.2.8]), and an Artinian reduction of $Z^{\prime}$ is a quotient of $R /\left(\mathcal{I}_{Z}+L\right)$.

Remark 28. Assume that $H_{Z}(d)=\ell_{Z}$. Then the map $\rho_{d}: H^{0}(\mathcal{O}(d)) \rightarrow H^{0}\left(\mathcal{O}_{Z}\right)$ introduced in Remark 22 surjects. Thus all the elements of $H^{0}\left(\mathcal{O}_{Z}\right) \simeq \mathbb{C}^{\ell_{Z}}$ lives in the image of the evaluation map. In particular, the vector $\left[\begin{array}{llll}1 & 0 & \cdots & 0\end{array}\right]$ is in the image. This implies that there is a form $f$ of degree $d$ vanishing at all the points of $Z$ except for the first one. Geometrically this means that there exists a hypersurface of degree $d$ in $\mathbb{P C}^{n+1}$ that contains all but one points of $Z$. As the same phenomenon occurs for all elements of the natural basis of $H^{0}\left(\mathcal{O}_{Z}\right)=\mathbb{C}^{\ell_{Z}}$, we can find for every $P \in Z$ a hypersurface of degree $d$ that contains $Z \backslash\{P\}$ and excludes $P$. Thus, if $H_{Z}(d)=\ell_{Z}$, then we will say that hypersurfaces of degree $d$ separate the points of $Z$.

The Hilbert function is closely related to the linear properties of the images of $Z$ under Veronese maps of increasing degrees.

Proposition 29. $H_{Z}(d)$ is equal to the (projective) dimension of the linear span of the image of $Z$ in $v_{d}$ plus $1: H_{Z}(d)=\operatorname{dim}\left\langle v_{d}(Z)\right\rangle+1$. Consequently, $H_{Z}(d)=\ell_{Z}$ if and only if the points of $v_{d}(Z)$ are linearly independent.

Proof. The projective dimension $\delta$ of the linear span $\left\langle v_{d}(Z)\right\rangle$ is equal to $N$ minus the affine dimension of the space of linear forms whose corresponding hyperplanes in 
$\mathbb{P} \mathbb{C}^{N+1}$ contain $v_{d}(Z)$. Thus $\delta$ is equal to $N-\operatorname{dim}\left(J_{1}\right)$, where $J$ is the homogeneous ideal of $v_{d}(Z)$ in $\mathbb{P} \mathbb{C}^{N+1}$ and $J_{1}$ denotes the linear forms of $J$. Now notice that $N+1=\left(\begin{array}{c}n+d \\ d\end{array}\right)=\operatorname{dim} R_{d}$. Moreover, $J_{1}$ corresponds to the space of forms in $R_{d}$ which contain $Z$. Since, by definition, $H_{Z}(d)=\operatorname{dim} R_{d}-\operatorname{dim} I_{d}$, where $I \subset R$ is the homogeneous ideal of $Z$ in $\mathbb{P} \mathbb{C}^{n+1}$, the claim follows.

If $Z$ is the union of two disjoint sets $A$ and $B$, then the Hilbert function provides a way to compute the dimension of the intersection $\left\langle v_{d}(A)\right\rangle \cap\left\langle v_{d}(B)\right\rangle$.

Proposition 30. If $A$ and $B$ are subsets of $\mathbb{P} \mathbb{C}^{n+1}$ and both $v_{d}(A)$ and $v_{d}(B)$ are linearly independent sets, then $\operatorname{dim}\left(\left\langle v_{d}(A)\right\rangle \cap\left\langle v_{d}(B)\right\rangle\right)=\ell_{A}+\ell_{B}-H_{Z}(d)-1$, where $Z=A \cup B$.

Proof. We use the Grassmann formula:

$\operatorname{dim}\left(\left\langle v_{d}(A)\right\rangle \cap\left\langle v_{d}(B)\right\rangle\right)=\operatorname{dim}\left(\left\langle v_{d}(A)\right\rangle\right)+\operatorname{dim}\left(\left\langle v_{d}(B)\right\rangle\right)-\operatorname{dim}\left(\left\langle v_{d}(A)\right\rangle+\left\langle v_{d}(B)\right\rangle\right)$.

Since $v_{d}(A)$ and $v_{d}(B)$ are linearly independent, it follows that $\operatorname{dim}\left(\left\langle v_{d}(A)\right\rangle\right)=\ell_{A}-1$ and $\operatorname{dim}\left(\left\langle v_{d}(B)\right\rangle\right)=\ell_{B}-1$. Moreover by Proposition 29, $\operatorname{dim}\left(\left\langle v_{d}(A)\right\rangle+\left\langle v_{d}(B)\right\rangle\right)=$ $\operatorname{dim}\left(\left\langle v_{d}(A) \cup v_{d}(B)\right\rangle\right)=H_{Z}(d)-1$. The claim follows.

We introduce a fundamental property of finite sets of points in a projective space.

DeFinition 31. We say that a finite set of points $Z \subset \mathbb{P} \mathbb{C}^{n+1}$ satisfies the CayleyBacharach property in degree $d$-abbreviated as $C B(d)$-if for every $P \in Z$ every form of degree $d$ vanishing at $Z \backslash\{P\}$ also vanishes at $P$.

If $Z$ satisfies $C B(d)$, then hypersurfaces of degree $d$ cannot separate the points of $Z$; in some sense $C B(P)$ is the exact opposite of separation. Thus, if $Z$ satisfies $C B(d)$, then $H_{Z}(d)<\ell_{Z}$ and $h_{Z}(d+1)>0$. However, the converse is false. For instance, the set $Z$ consisting of four points in $\mathbb{P} \mathbb{C}^{3}$, three of them aligned, does not satisfy $C B(1)$, while $H_{Z}(1)<4$.

The main reason for introducing the $C B(d)$ property is the following result, which strongly restricts the Hilbert functions of sets with a Cayley-Bacharach property.

Theorem 32 (Geramita, Kreuzer, and Robbiano [30]). The h-vector of a set of points $Z$ which satisfies $C B(d)$ has the following property: for all $k \geq 0$,

$$
h_{Z}(0)+h_{Z}(1)+\cdots+h_{Z}(k) \leq h_{Z}(d+1-k)+\cdots+h_{Z}(d)+h_{Z}(d+1) .
$$

We proceed by showing the link between Hilbert functions of finite sets and the identifiability problem for symmetric tensors. Let $\mathfrak{A} \in S^{d}\left(\mathbb{C}^{n+1}\right)$ be a symmetric tensor with two different "minimal" decompositions $\mathfrak{A}=\mathbf{v}_{1}^{\circ d}+\cdots+\mathbf{v}_{r}^{\circ d}=\mathbf{w}_{1}^{\circ d}+\cdots \mathbf{w}_{s}^{\circ d}$. In the present context, minimality of the decompositions means that $\mathfrak{A}$ does not lie in the span of a proper subset of the $\mathbf{v}_{i}^{o d}$ 's or of the $\mathbf{w}_{j}^{\circ d}$ 's. Let $P_{i}=\left[\mathbf{v}_{i}\right]$ and $Q_{j}=\left[\mathbf{w}_{j}\right]$ be the points of $\mathbb{P} \mathbb{C}^{n+1}$ corresponding to the elements of the decompositions. Define

$$
A=\left\{P_{1}, \ldots, P_{r}\right\}, \quad B=\left\{Q_{1}, \ldots, Q_{s}\right\}, \text { and } \quad Z=A \cup B .
$$

Then, the projective point $[\mathfrak{A}] \in \mathbb{P}\left(S^{d} \mathbb{C}^{n+1}\right)$ belongs to both spans $\left\langle v_{d}(A)\right\rangle$ and $\left\langle v_{d}(B)\right\rangle$. The minimality assumption means that $[\mathfrak{A}]$ does not belong to the linear span of any proper subset of either $v_{d}(A)$ or $v_{d}(B)$. So, the intersection $\left\langle v_{d}(A)\right\rangle \cap\left\langle v_{d}(B \backslash A)\right\rangle$

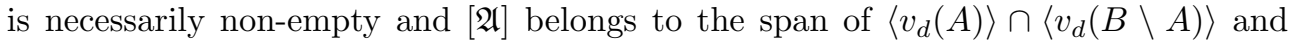
$v_{d}(A) \cap v_{d}(B)$. In particular, it follows that the points of $\left\langle v_{d}(Z)\right\rangle$ are not linearly independent. Hence $H_{Z}(d)<\ell(Z)$, so that $h_{Z}(d+1)>0$ by Proposition 26(iv). 
In applications, we are mainly confronted with sets $A$ and $B$ that are in GLP, essentially because of Lemma 12 . In terms of the Hilbert function, $Z$ is in GLP if and only if for every subset $Z^{\prime}$ of $Z$ of $k \leq n+1$ points we have that $H_{Z^{\prime}}(1)=k$ and $h_{Z^{\prime}}(1)=k-1$. In other words, if we consider an $(n+1) \times \ell_{Z}$ matrix $M$ whose columns consist of the projective coordinates for the points of $Z$, then $Z$ is in GLP if and only if every set of $\min \left\{\ell_{Z}, n+1\right\}$ columns of $M$ is linearly independent.

6. An effective criterion for $S^{4} \mathbb{C}^{4}$. We show how an analysis of the Hilbert function yields an effective criterion for symmetric tensors of type $4 \times 4 \times 4 \times 4$. The goal consists of affirming the $r$-identifiability of a tensor

$$
\mathfrak{A}=\mathbf{v}_{1}^{\circ 4}+\cdots+\mathbf{v}_{r}^{\circ 4}
$$

for any value of $r$. The results of [23,42] entail that generic tensors of rank $r=8$ in $\mathbb{P}\left(S^{4} \mathbb{C}^{4}\right)$ are (exceptionally) not 8-identifiable; they admit two distinct complex decompositions; see, e.g., [22, Section 2]. Consequently, decompositions with $r \geq 9$ are also not generically $r$-identifiable. On the other hand, it was proved in [6] that generic tensors of rank $r \leq 7$ in $\mathbb{P}\left(S^{4} \mathbb{C}^{4}\right)$ are identifiable. ${ }^{6}$ An effective criterion for $4 \times 4 \times 4 \times 4$ symmetric tensors should thus certify generic $r$-identifiability for all $r \leq 7$. The reshaped Kruskal criterion (Corollary 20) is effective in the symmetric setting if $r \leq\left(\begin{array}{c}3+2 \\ 2\end{array}\right)+\min \left\{\frac{1}{2} \delta, \delta\right\}=10-4=6$ because $\delta=4+4-\left(\begin{array}{c}3+2 \\ 2\end{array}\right)-2=-4$. To our knowledge, no effective criterion is known for $r=7$ in the literature. Here we derive such a criterion, thereby concluding the proof of Theorem 2.

6.1. Theory. Assume that we are given the decomposition (7) with length $r=7$ and that we should determine if $\mathfrak{A}$ is 7 -identifiable. We make two assumptions. First, we assume that the given decomposition is minimal. One can ascertain minimality by checking that $H_{A}(4)=7$, which is a rank computation. If the decomposition is not minimal, then it is not of rank 7 , and so not 7 -identifiable. The second assumption is that $A=\left\{\left[\mathbf{v}_{1}\right], \ldots,\left[\mathbf{v}_{7}\right]\right\}$ is in GLP, a condition which is easily verified. By Lemma 12 , the subset of points not in GLP on $\sigma_{7}\left(v_{4}\left(\mathbb{C}^{4}\right)\right)$ forms a Zariski-closed set. Hence, this assumption will not alter the effectiveness of our criterion.

We show that a different decomposition $\mathfrak{A}=\mathbf{w}_{1}^{\circ 4}+\cdots+\mathbf{w}_{s}^{\circ 4}$ with $s \leq 7$ does not exist. Arguing by contradiction, we assume that a second decomposition exists and investigate which consequences it has on the geometry of $A$. We can assume without loss of generality that this alternative decomposition is minimal. In the remainder, let $B=\left\{\left[\mathbf{w}_{1}\right], \ldots,\left[\mathbf{w}_{s}\right]\right\}$ and $Z=A \cup B$.

Proposition 33. If alternative decompositions exist, then we can choose an alternative decomposition with $A$ and $B$ disjoint.

The proof of this result is delayed until after Proposition 35.

Proposition 34. Alternative decompositions exist only if $Z$ satisfies $C B(4)$.

Proof. Assume it does not. Then, there exist a $P \in Z$ and a form of degree 4 that contains $Z^{\prime}=Z \backslash\{P\}$ but excludes $P$. Thus, the homogeneous ideals satisfy $\operatorname{dim}\left(I_{Z}\right)_{4}<\operatorname{dim}\left(I_{Z^{\prime}}\right)_{4}$, so that $H_{Z}(4)>H_{Z^{\prime}}(4)$. It follows that $h_{Z}(q)>h_{Z^{\prime}}(q)$ for some value $q \leq 4$. Since $h_{Z}(i) \geq h_{Z^{\prime}}(i)$ for all $i$ by Proposition 27 , and $\sum h_{Z}(i)=$ $\ell_{Z}=1+\ell_{Z^{\prime}}=1+\sum h_{Z^{\prime}}(i)$, it follows that $h_{Z}(q)=1+h_{Z^{\prime}}(q)$ and $h_{Z}(i)=h_{Z^{\prime}}(i)$ for $i \neq q$. Thus, $H_{Z}(4)=H_{Z^{\prime}}(4)+1$.

\footnotetext{
${ }^{6}$ Combining the Alexander-Hirschowitz theorem [2] with [42, Corollary 4.5] also yields this result.
} 
Now assume that $P \in A$ and recall that we may assume $A \cap B=\emptyset$ by Proposition 33. Setting $A^{\prime}=A \backslash\{P\}$, we get from Proposition 30 that

$$
\begin{aligned}
\operatorname{dim}\left(\left\langle v_{4}(A)\right\rangle \cap\left\langle v_{4}(B)\right\rangle\right)=\ell_{A}+\ell_{B}-H_{Z}(4)-1 & =\ell_{A^{\prime}}+\ell_{B}-H_{Z^{\prime}}(4)-1 \\
& =\operatorname{dim}\left(\left\langle v_{4}\left(A^{\prime}\right)\right\rangle \cap\left\langle v_{4}(B)\right\rangle\right),
\end{aligned}
$$

so that $\left\langle v_{4}(A)\right\rangle \cap\left\langle v_{4}(B)\right\rangle=\left\langle v_{4}\left(A^{\prime}\right)\right\rangle \cap\left\langle v_{4}(B)\right\rangle$. Consequently, $\mathfrak{A}$ belongs to $v_{4}\left(A^{\prime}\right)$, contradicting the assumption of minimality. If $P \in B$ we similarly obtain that $\mathfrak{A}$ belongs to the span of $v_{4}(B \backslash\{P\})$, contradicting the minimality of $B$.

Proposition 35. Alternative decompositions exist only if $s=|B|=7$. The $h$ vector of $Z$ is $(1,3,3,3,3,1)$ and $Z$ is contained in an irreducible twisted cubic curve.

Proof. Since $A$ is in GLP, the $h$-vector of $A$ is $(1,3,3)$. Indeed, $h_{A}(0)=1$ is obvious, while $h_{A}(1)=3$ because $A$ spans $\mathbb{P C}^{4}$. So by Proposition 26 it just remains to prove that $H_{A}(2)=7$. For any $P \in A$, divide the remaining 6 points in two set of three points each, and then take the two planes spanned by the two sets. As $A$ is in GLP, no four points of $A$ belong to a plane, so that the two planes define a quadric that contains $A \backslash\{P\}$ and misses $P$. Thus, $A$ is separated by quadrics and $H_{A}(2)=7$ by Remark 28 .

$Z$ satisfies $C B(4)$ by Proposition 34 , and hence, by Theorem 32,

$$
\begin{aligned}
h_{Z}(5) & \geq h_{Z}(0)=1 \\
h_{Z}(4)+h_{Z}(5) & \geq h_{Z}(0)+h_{Z}(1)=4, \text { and } \\
h_{Z}(3)+h_{Z}(4)+h_{Z}(5) & \geq h_{Z}(0)+h_{Z}(1)+h_{Z}(2)=4+h_{Z}(2) .
\end{aligned}
$$

Since $h_{Z}(2) \geq h_{A}(2)=3$ by Proposition $27, \ell_{Z} \geq \sum_{i=0}^{5} h_{Z}(i) \geq 14$ so that $s \geq 7$. It follows that $s=7$ and $\ell_{Z}=\sum_{i=0}^{5} h_{Z}(i)=H_{Z}(5)=14$, and, hence, $h_{Z}(2)=3$. In particular $H_{Z}(2)=7$, so $Z$ is contained in three linearly independent quadric surfaces. Clearly these quadric surfaces cannot meet in a finite number of points, since $\ell_{Z}>8$. We will prove that $C$ is a twisted cubic curve that contains $Z$.

Notice that $h_{Z}(3)$ cannot be bigger that 3 , because $h_{Z}(4)+h_{Z}(5) \geq 4$. If $h_{Z}(3) \leq$ 2 , then by [12, Theorem 3.6] and its proof one has also $h_{Z}(4), h_{Z}(5) \leq 2$, contradicting $h_{Z}(3)+h_{Z}(4)+h_{Z}(5) \geq h_{Z}(2)+h_{Z}(1)+h_{Z}(0)=7$. Hence $h_{Z}(3)=3$. It also follows that $h_{Z}(4)+h_{Z}(5) \leq 4$. Thus, equality holds. If $h_{Z}(4) \leq 1$ then also $h_{Z}(5) \leq 1$ by $[12$, Theorem 3.6] again, which is a contradiction. Hence, there are only two possibility left for the $h$-vector of $Z$, namely $h_{Z}=(1,3,3,3,2,2)$ or $h_{Z}=(1,3,3,3,3,1)$. Next, we use again [12, Theorem 3.6]. In the former case, since $h_{Z}(4)=h_{Z}(5)=2$, then there exists a curve $C$ of degree 2 containing a subset $Z^{\prime} \subset Z$, and the ideal of $C$ coincides with the ideal of $Z^{\prime}$ up to degree 5 . If $C$ is a conic, then it must contain at least 11 points of $Z^{\prime}$, hence at least 4 points of $A$, which is impossible since a conic is a plane curve and $A$ is in GLP. If $C$ is a disjoint union of lines then it must contain at least 12 points of $Z$, hence at least 5 points of $A$, which is excluded since $A$ has no three points on a line.

We can conclude that the $h$-vector of $Z$ is $(1,3,3,3,3,1)$, so $h_{Z}(3)=h_{Z}(4)=3$. Then, by [12, Theorem 3.6] there exists a cubic curve $C$ which contains a subset $Z^{\prime}$ of $Z$ whose ideal coincides with the ideal of $C$ up to degree 4 . If $C$ is a plane curve, then its $h$-vector is $(1,2,3,3,3, \ldots)$, so $Z^{\prime}$ can miss at most 2 points of $Z$, which contradicts again the GLP of $A$. If $C$ spans $\mathbb{P C}^{4}$, then the $h$-vector of $C$ is $(1,3,3,3,3, \ldots)$ and the homogeneous ideal is generated in degree at most 3 . So, if $C$ misses some points of $Z$, then $h_{Z}(3)>h_{C}(3)=3$, which is a contradiction. Thus $C$ contains $Z$, hence it contains $A$. 
It remains to show that $C$ is irreducible. $C$ cannot split in three lines, for one line would then contain three points of $A$. If it splits in a line and an irreducible (plane) conic, then either there exists a line containing three points of $A$, or 5 points of $A$ lie in the plane of the conic. Both situations contradict the GLP of $A$.

Proof of Proposition 33. Suppose that in every alternative decomposition $B$ of cardinality equal to the rank $s \leq 7$ of $\mathfrak{A}$ some of the points appear in both $A$ and $B$, say $A \cap B=\left\{\left[\mathbf{v}_{1}\right], \ldots,\left[\mathbf{v}_{k}\right]\right\}$ with $k>0$. Then

$$
\mathfrak{A}=\mathbf{v}_{1}^{\circ 4}+\mathbf{v}_{2}^{\circ 4}+\cdots+\mathbf{v}_{7}^{\circ 4}=\lambda_{1} \mathbf{v}_{1}^{\circ 4}+\cdots+\lambda_{k} \mathbf{v}_{k}^{\circ 4}+\mathbf{w}_{k+1}^{\circ 4}+\cdots+\mathbf{w}_{s}^{\circ 4} .
$$

It follows that

$$
\mathfrak{A}^{\prime}=\left(1-\lambda_{1}\right) \mathbf{v}_{1}^{\circ 4}+\cdots+\left(1-\lambda_{k}\right) \mathbf{v}_{k}^{\circ 4}+\mathbf{v}_{k+1}^{\circ 4}+\cdots+\mathbf{v}_{7}^{\circ 4}=\mathbf{w}_{k+1}^{\circ 4}+\cdots+\mathbf{w}_{s}^{\circ 4} .
$$

If any of the $\lambda_{j}$ are equal to 1 , then $\mathfrak{A}^{\prime}$ would be an identifiable tensor because of Kruskal's theorem and the assumption that $A$ is in GLP. It follows that $s \geq 7$, hence, $s=7$. Comparing the lengths of the decompositions of $\mathfrak{A}^{\prime}$, it follows that all $\lambda_{j}=1$. But then $\left\{\left[\mathbf{v}_{k+1}\right], \ldots,\left[\mathbf{v}_{7}\right]\right\}=\left\{\left[\mathbf{w}_{k+1}\right], \ldots,\left[\mathbf{w}_{7}\right]\right\}$ because of the identifiability of $\mathfrak{A}^{\prime}$. This implies the decompositions $A$ and $B$ of $\mathfrak{A}$ consist of the same set of points: $A=B$. By the assumption on minimality of $A$, it follows that $\mathfrak{A}$ is identifiable as well, which contradicts our assumption.

So, none of the $\lambda_{j}$ are equal to 1 . Then $\mathfrak{A}^{\prime}$ has two decompositions, $A$ is still in GLP, and we let $B^{\prime}=B \backslash A=\left\{\left[\mathbf{w}_{k+1}\right], \ldots,\left[\mathbf{w}_{s}\right]\right\}$ and $Z^{\prime}=A \cup B^{\prime}$. Applying Proposition 35 to $Z^{\prime}$ yields that $\mathfrak{A}^{\prime}$ has alternative decompositions only if $\left|B^{\prime}\right|=7$, requiring $s \geq 8 \not \leq 7$, contradicting the assumption that $B$ was of minimal cardinality.

This proves that if $\mathfrak{A}$ is not 7-identifiable with $A$ in GLP, then there must exist at least one set of points $B$ such that $A \cap B=\emptyset$ and $\mathfrak{A} \in\left\langle v_{d}(A)\right\rangle \cap\left\langle v_{d}(B)\right\rangle$.

Proposition 36. If $A$ is contained in an irreducible rational twisted cubic curve $C$, then $\mathfrak{A}$ is not identifiable and the given decomposition of $\mathfrak{A}$ is contained in a positive dimensional family of decompositions. In other words, there exists a positive dimensional family of subsets $A_{t}$ of cardinality 7 in $v_{4}\left(\mathbb{P C}^{4}\right)$, with $A_{0}=A$, such that $\mathfrak{A}$ belongs to the span of each $v_{4}\left(A_{t}\right)$.

Proof. The twisted cubic is itself the image of a Veronese map $C=v_{3}\left(\mathbb{P} \mathbb{C}^{2}\right)$, thus $v_{4}(C)=v_{12}\left(\mathbb{P C}^{2}\right)$ is a rational normal curve in $\mathbb{P C}^{13}$. The secant variety $\sigma_{7}\left(v_{12}\left(\mathbb{P C}^{2}\right)\right)$ covers $\mathbb{P} \mathbb{C}^{13}$ and every rank-7 point of $\mathbb{P} \mathbb{C}^{13}$ is contained in a 1-dimensional family of 7 secant spaces. Thus when $A$ is contained in a twisted cubic, then $\mathfrak{A}$ lies into the space $\mathbb{P} \mathbb{C}^{13}$ spanned by $v_{4}(C)=v_{12}\left(\mathbb{P C}^{2}\right)$ and consequently it has infinitely many decompositions as a sum of 7 tensors of rank 1, lying in $v_{4}(C)$. Thus there exists a 1-dimensional family of decompositions for $\mathfrak{A}$ which includes $A$.

Verifying that there does not exist a positive dimensional family of alternative decompositions over $\mathbb{F}$ may be accomplished by exploiting the following result, which is essentially implicit in Terracini's paper [55].

Lemma 37. Let $\mathcal{V} \subset \mathbb{F}^{N}$ be an affine variety that is not $r$-defective. Let the points $p_{1}, \ldots, p_{r} \in \mathcal{V}$, and let $\mathrm{T}_{p_{i}} \mathcal{V} \subset \mathbb{F}^{N}$ denote the affine tangent space to $\mathcal{V}$ at $p_{i}$. If the $p_{i}$ 's are contained in a family of decompositions of positive dimension, then $\operatorname{dim}\left\langle\mathrm{T}_{p_{1}} \mathcal{V}, \ldots, \mathrm{T}_{p_{r}} \mathcal{V}\right\rangle<\operatorname{dim} \sigma_{r}(\mathcal{V})$.

Proof. Let $p=\sum_{i=1}^{r} p_{i}(t)$ with $p_{i}(0)=p_{i}$ and $t$ in a neighborhood of zero be a smooth curve passing through the $p_{i}$ 's along which $p$ remains constant. As $\mathcal{V}$ is a variety, the Taylor series expansion of this analytic curve is well-defined and 
by [39, Lemma 2.1] may be written as $p_{i}(t)=p_{i}+t p_{i}^{(1)}+t^{2} p_{i}^{(2)}+\cdots$ with $p_{i}^{(1)} \in \mathrm{T}_{p_{i}} \mathcal{V}$ and $p_{i}^{(k)} \in \mathbb{F}^{N}$. After grouping terms by powers of $t$, we have

$$
p=p+t \sum_{i=1}^{r} p_{i}^{(1)}+t^{2} \sum_{i=1}^{r} p_{i}^{(2)}+\cdots
$$

Since this holds for all $t$ in a neighborhood of 0 , it follows that $\sum_{i=1}^{r} p_{i}^{(k)}=0$ for all $k$. In particular the case $k=1$ entails that $\operatorname{dim}\left\langle\mathrm{T}_{p_{1}} \mathcal{V}, \ldots, \mathrm{T}_{p_{r}} \mathcal{V}\right\rangle$ is strictly less than the expected dimension of $\sigma_{r}(\mathcal{V})$. By assumption on $\mathcal{V}$, this concludes the proof.

By Terracini's Lemma [55] we know that if the $\left(p_{1}, \ldots, p_{r}\right)$ are generic and $\mathcal{V}$ is nondefective, then $\operatorname{dim}\left\langle\mathrm{T}_{p_{1}} \mathcal{V}, \ldots, \mathrm{T}_{p_{r}} \mathcal{V}\right\rangle=\operatorname{dim} \sigma_{r}(\mathcal{V})$ so that the foregoing lemma can effectively exclude the possibility that such a positive dimensional family exists. The next sufficient condition for specific 7-identifiability in $S^{4} \mathbb{C}^{4}$ is then obtained.

Proposition 38. Let $\mathbb{F}=\mathbb{R}$ or $\mathbb{C}$. Let $p_{i}=\lambda_{i} \mathbf{a}_{i}^{\circ 4} \in v_{4}\left(\mathbb{F}^{4}\right) \subset S^{4} \mathbb{F}^{4}$, with $\lambda_{i} \in \mathbb{F}$ and $\mathbf{a}_{i} \in \mathbb{F}^{4}$ for $i=1, \ldots, 7$, be given in the form of a factor matrix $A=$ $\left[\lambda_{1}^{1 / 4} \mathbf{a}_{1} \cdots \lambda_{7}^{1 / 4} \mathbf{a}_{7}\right]$. If $A$ is in $G L P, A \odot A \odot A \odot A$ is of rank 7 , and there does not exist a family of alternative complex decompositions passing through $A$, then $\mathfrak{A}=$ $\sum_{i=1}^{7} \lambda_{i} \mathbf{a}_{i}^{\circ 4} \in S^{4} \mathbb{F}^{4}$ is 7 -identifiable over $\mathbb{C}$, and, hence, 7 -identifiable over $\mathbb{F}$.

Proof. For $\mathbb{F}=\mathbb{C}$, we can assume without loss of generality that all $\lambda_{i}=1$. The result then follows from Proposition 36.

For $\mathbb{F}=\mathbb{R}$, it suffices to note that we can apply Proposition 36 to every complex decomposition of length 7 , in particular we can apply it to the right-hand side of

$$
\mathfrak{A}=\sum_{i=1}^{7} \lambda_{i} \mathbf{a}_{i}^{\circ 4}=\sum_{i=1}^{7}\left(\lambda_{i}^{1 / 4} \mathbf{a}_{i}\right)^{\circ 4} \in S^{4} \mathbb{R}^{4}
$$

which in general is a complex Waring decomposition. If the conditions of the proposition are satisfied for the decomposition on the right-hand side, then it also proves that the corresponding real Waring decomposition is the unique complex decomposition of $\mathfrak{A}$, and, hence, it is the unique decomposition over both $\mathbb{R}$ and $\mathbb{C}$.

6.2. The algorithm. Assume that we are given a decomposition

$$
\mathfrak{A}=\sum_{i=1}^{r} p_{i}=\sum_{i=1}^{r} \lambda_{i} \mathbf{a}_{i}^{\circ 4} \in S^{4} \mathbb{F}^{4}
$$

with $\lambda_{i} \in \mathbb{F}_{0}$ and $\mathbf{a}_{i} \in \mathbb{F}^{4} \backslash\{0\}, i=1, \ldots, r$, as a matrix $A=\left[\lambda_{1}^{1 / 4} \mathbf{a}_{1} \cdots \lambda_{1}^{1 / 4} \mathbf{a}_{r}\right] \in \mathbb{C}^{4 \times r}$. Then, the following steps should be taken.

S1. If $r \geq 8$, the algorithm terminates claiming that it can not prove the identifiability of $\mathfrak{A}$.

S2. If $r=1$, the algorithm terminates, stating that $\mathfrak{A}$ is 1-identifiable.

S3. If $2 \leq r \leq 6$, perform the following steps:

S3a. Compute the Kruskal ranks $\kappa_{1}$ and $\kappa_{2}$ of $A$ and $A \odot A$ respectively.

S3b. If $r \leq \kappa_{1}+\frac{1}{2} \kappa_{2}-1$, then the algorithm terminates stating that $\mathfrak{A}$ is $r$-identifiable. Otherwise it terminates, unable to prove identifiability.

S4. If $r=7$, perform the following steps:

S4a. Compute $A \odot A \odot A \odot A$ and verify that its rank equals 7 . If it does not, the algorithm terminates stating that $\mathfrak{A}$ is not 7 -identifiable.

S4b. Compute the Kruskal rank of $A$. If it is not 4, the algorithm terminates claiming that it cannot prove identifiability. 
S4c. Let $T_{i}$ be a basis for the tangent space $\mathrm{T}_{p_{i}} v_{4}\left(\mathbb{C}^{4}\right)$. Compute the rank of $T=\left[\begin{array}{lll}T_{1} & \cdots & T_{r}\end{array}\right]$. If it does not equal 21 , then the algorithm terminates claiming that it cannot prove 7 -identifiability.

S4d. The algorithm terminates, stating that $\mathfrak{A}$ is 7-identifiable.

Proof of Theorem 2. The fact that the above algorithm is effective for all tensors in $S^{4} \mathbb{F}^{4}$ is trivial for $r=1$; it follows from Corollary 20 for $2 \leq r \leq 6$; for $r=7$ it follows from the fact that the assumptions leading to Proposition 36, namely GLP and minimality, fail only on Zariski-closed sets as well as the fact that $\mathbb{P} v_{4}\left(\mathbb{C}^{4}\right)$ is not defective for $r=7$ [2] so that the dimension condition in Lemma 37 is only satisfied on a Zariski-closed set - effectiveness in the real case follows from the foregoing, [47] and the fact that $\mathbb{P} v_{4}\left(\mathbb{C}^{4}\right)$ is not 7-defective; and for $r \geq 8$ the generic element of $\sigma_{r}(\mathcal{V})$ is not complex $r$-identifiable.

6.3. Two examples. We present two cases illustrating the foregoing algorithm in the original case $r=7$ using our implementation in Macaulay2 [32]. ${ }^{7}$

An identifiable example. Consider a real Waring decomposition of length 7 that was randomly generated in Macaulay2:

$$
\mathfrak{A}=\sum_{i=1}^{7} \mathbf{a}_{i}^{\circ 4}, \quad \text { with } A=\left[\mathbf{a}_{i}\right]_{i=1}^{7}=\left[\begin{array}{ccccccc}
5 & -3 & 1 & 7 & 3 & 1 & -9 \\
0 & 9 & 1 & 2 & 8 & -2 & 6 \\
-8 & 5 & 5 & -3 & -4 & -6 & -8 \\
3 & 7 & 9 & -3 & 8 & 7 & -7
\end{array}\right]
$$

Executing the algorithm, we can skip steps S1-S3 and immediately move to step S4a. The rank of $A \odot A \odot A \odot A$ is computed to be 7 , so we proceed with step S4b. The Kruskal rank of $A$, which consists of computing the rank of $357 \times 7$ matrices, is computed to be 4 . In step S4c, we compute rank of the $35 \times 28$ matrix $T$ whose columns span a subspace of the tangent space to $\sigma_{r}\left(\mathcal{S}_{\mathbb{C}}\right)$ at $\mathfrak{A}$. The rank of this matrix is the maximal value 28, so by Proposition 38 we may conclude that there is just one complex Waring decomposition. Since we started from a real decomposition, it follows that this is the unique Waring decomposition of $\mathfrak{A}$.

A nonidentifiable example. The following classical lemma gives infinitely many Waring decompositions of the degree 12 binary form $\left(x^{2}+y^{2}\right)^{6}$. The seven summands correspond to seven consecutive vertices of a regular 14-gon in the Euclidean plane with coordinates $(x, y)$.

Lemma 39 (Reznick [48, Theorem 9.5]). Let $R=2^{-12} \cdot 7 \cdot\left(\begin{array}{c}12 \\ 6\end{array}\right)$. Then $\forall \phi \in \mathbb{R}$ :

$$
\sum_{k=0}^{6}\left(\cos \left(\frac{k \pi}{7}+\phi\right) x+\sin \left(\frac{k \pi}{7}+\phi\right) y\right)^{12}=R\left(x^{2}+y^{2}\right)^{6}
$$

These decompositions are minimal, in the sense that $\operatorname{rank}_{\mathbb{C}}\left(\left(x^{2}+y^{2}\right)^{6}\right)=7$.

From the previous lemma we get the following example with infinitely many decompositions of a rank 7 symmetric tensor in $\mathbb{R}^{4} \otimes \mathbb{R}^{4} \otimes \mathbb{R}^{4} \otimes \mathbb{R}^{4}$. Let $z_{0}, \ldots, z_{3}$ be coordinates in $\mathbb{R}^{4}$ and let $A_{k, \phi}=\cos ^{3}\left(\frac{k \pi}{7}+\phi\right) z_{0}+\cos ^{2}\left(\frac{k \pi}{7}+\phi\right) \sin \left(\frac{k \pi}{7}+\phi\right) z_{1}+$ $\cos \left(\frac{k \pi}{7}+\phi\right) \sin ^{2}\left(\frac{k \pi}{7}+\phi\right) z_{2}+\sin ^{3}\left(\frac{k \pi}{7}+\phi\right) z_{3}$ be a linear form in $\mathbb{P R}^{4}$. These linear forms correspond to points on the twisted cubic curve parametrized by $z_{i}=x^{3-i} y^{i}$

\footnotetext{
${ }^{7}$ Our implementation in Macaulay2 can be accessed at https://arxiv.org/abs/1609.00123.
} 


$$
\mathfrak{A}=\sum_{k=0}^{6} \mathbf{a}_{k, \phi}^{\circ 4} \quad \text { with } \mathbf{a}_{k, \phi}=\left[\begin{array}{c}
\cos ^{3}\left(\frac{k \pi}{7}+\phi\right) \\
\cos ^{2}\left(\frac{k \pi}{7}+\phi\right) \sin \left(\frac{k \pi}{7}+\phi\right) \\
\cos \left(\frac{k \pi}{7}+\phi\right) \sin ^{2}\left(\frac{k \pi}{7}+\phi\right) \\
\sin ^{3}\left(\frac{k \pi}{7}+\phi\right)
\end{array}\right]
$$

Then $\mathfrak{A}$ is a symmetric tensor in $\mathbb{R}^{4} \otimes \mathbb{R}^{4} \otimes \mathbb{R}^{4} \otimes \mathbb{R}^{4}$ (or equivalently a quartic polynomial) which does not depend on $\phi$ by Lemma 39. For every $\phi,(8)$ is a different Waring decomposition with seven summands of $\mathfrak{A}$.

We now apply the algorithm to this example, where we have chosen $\phi=0$ as particular decomposition to be handed to the algorithm. It will be necessary to perform numerical computations as $\mathfrak{A}$ no longer admits coordinates over the integers. The $\epsilon$-rank of a matrix is defined as the number of singular values that are larger than $\epsilon$; the rank of a matrix is its 0 -rank. There always exists a positive $\delta>0$ such that all the $\delta^{\prime}$-ranks of a matrix are equal for all $0 \leq \delta^{\prime} \leq \delta$. Through a rigorous perturbation analysis this value of $\delta$ can be determined; however, in this brief example such an approach is not pursued. In Macaulay2, the $\epsilon$-rank can be computed with the numericalRank function from the NumericalAlgebraicGeometry package. In our experiment, we used the arbitrary choice $\epsilon=10^{-12}$. Running the algorithm, it skips steps S1, S2 and S3. The numerical rank of $A \odot A \odot A \odot A$ was determined to be 7 in step S4a (the largest and smallest singular values were approximately 1.08615 and 0.21978 respectively). In step S4b, the (numerical) Kruskal rank was 4. Computing the singular values of $T$ in step S4c resulted in the following values:

$\begin{array}{cclllll}27.4692 ; & 27.3073 ; & 8.70636 ; & 8.59365 ; & 7.26970 ; & 7.11095 ; & 7.02903 ; \\ 6.83427 ; & 4.05864 ; & 3.89601 ; & 3.01363 ; & 2.45649 ; & 2.24154 ; & 2.07335 ; \\ 1.90712 ; & 1.90496 ; & 1.58224 ; & 1.52450 ; & 1.35632 ; & 1.26918 ; & 1.00762 ; \\ 0.553879 ; & 0.481666 ; & 0.424916 ; & 0.364948 ; & 0.175228 ; & 0.165698 ; & 6.60364 \cdot 10^{-16} .\end{array}$

The numerical rank is only $27<\operatorname{dim} \sigma_{7}\left(v_{4}\left(\mathbb{C}^{4}\right)\right)=28$. So the algorithm terminates claiming that it cannot prove 7 -identifiability of $\mathfrak{A}=\sum_{k=0}^{6} \mathbf{a}_{k, 0}^{\circ 4}$. As $\mathfrak{A}$ has a family of decompositions of positive dimension, this was expected.

7. Application to algorithm design. An important consequence of Theorem 14 is that it provides a solid theoretical foundation for algorithms computing tensor rank decompositions based on reshaping, such as $[11,46]$. These algorithms attempt to recover a tensor rank decomposition of a rank- $r$ tensor as in (1), living in $\mathbb{F}^{n_{1}} \otimes \mathbb{F}^{n_{2}} \otimes$ $\cdots \otimes \mathbb{F}^{n_{d}}$, by considering $\mathfrak{A}$ as an element of $\mathbb{F}^{\Pi_{\mathbf{h}}} \otimes \mathbb{F}^{\Pi_{\mathbf{k}}} \otimes \mathbb{F}^{\Pi_{1}}$ with $\mathbf{h} \sqcup \mathbf{k} \sqcup \mathbf{l}=\{1,2, \ldots, d\}$ and instead computing a decomposition

$$
\mathfrak{A}=\sum_{i=1}^{r} \mathbf{b}_{i}^{1} \otimes \mathbf{b}_{i}^{2} \otimes \mathbf{b}_{i}^{3} .
$$

If both decompositions (1) and (9) are unique, then the rank-1 tensors satisfy $\mathbf{b}_{\sigma_{i}}^{1}=\mathbf{a}_{i}^{h_{1}} \otimes \mathbf{a}_{i}^{h_{2}} \otimes \cdots \otimes \mathbf{a}_{i}^{h_{s}}, \mathbf{b}_{\sigma_{i}}^{2}=\mathbf{a}_{i}^{k_{1}} \otimes \mathbf{a}_{i}^{k_{2}} \otimes \cdots \otimes \mathbf{a}_{i}^{k_{t}}, \quad$ and $\mathbf{b}_{\sigma_{i}}^{3}=\mathbf{a}_{i}^{l_{1}} \otimes \mathbf{a}_{i}^{l_{2}} \otimes \cdots \otimes \mathbf{a}_{i}^{l_{u}}$,

for some permutation $\sigma$ of $\{1,2, \ldots, r\}$, and where $s, t$, and $u$ are the cardinalities of $\mathbf{h}, \mathbf{k}$, and $\mathbf{l}$ respectively. One of the advantages of this approach is that decomposition (9) could be computed using one of the direct methods that exist only ${ }^{8}$ for third-order

\footnotetext{
${ }^{8}$ The algorithm by Bernardi, Brachat, Comon, and Mourrain [10] can compute a tensor rank decomposition of any tensor, but in general it requires the solution of a system of linear, quadratic and cubic equations.
} 
tensors, e.g., $[25,27,41]$. Thereafter, decomposition (1) can be efficiently recovered by computing rank- 1 decompositions of the vectors $\mathbf{b}_{i}^{k}$ for all $k=1,2,3$ and $i=$ $1,2, \ldots, r$, using one of several suitable algorithms, such as $[44,49,56,57]$.

The conditions under which aforementioned algorithms are expected to recover the decomposition (1) have not been studied. This is precisely the problem that Lemma 12 and Theorem 14 tackle: if (1) is a generic decomposition with $r$ satisfying bound (6), then decompositions (1) and (9) are simultaneously unique in the spaces $\mathbb{F}^{n_{1}} \otimes \cdots \otimes \mathbb{F}^{n_{d}}$ and $\mathbb{F}^{\Pi_{\mathbf{h}}} \otimes \mathbb{F}^{\Pi_{\mathbf{k}}} \otimes \mathbb{F}^{\Pi_{1}}$ respectively, entailing that the aforementioned reshaping-based algorithms can recover the unique decomposition (1) of $\mathfrak{A}$ via (9).

8. Conclusions. An important quality measure for criteria for identifiability of tensors is its effectiveness. Theorem 14 proves that the popular Kruskal criterion when it is combined with reshaping is effective. The proof yielded insight into reshapingbased algorithms for computing tensor rank decompositions, proving that they will recover the unique decomposition with probability 1 if the rank is within the range of effectiveness of Theorem 14. The range of effectiveness for symmetric identifiability of the reshaped Kruskal criterion was established. Combining this result with results from the literature established that a small number of low-dimensional symmetric tensor spaces are completely effectively identifiable. By analyzing the Hilbert function, we could prove complete effective identifiability of an additional case, namely $S^{4} \mathbb{F}^{4}$. Theorem 2 lists all known instances of completely effectively identifiable spaces.

All criteria for specific $r$-identifiability that we are aware of for order- $d$ tensors are applicable for $r$ up to about $\mathcal{O}\left(n^{d / 2}\right)$ when $n_{1}=\cdots=n_{d}=n$, whereas generic $r$-identifiability is expected to hold up to $\mathcal{O}\left(n^{d-1}\right)$. We believe that this gap is related to the fact that nonidentifiable points on a generically $r$-identifiable variety where Terracini's matrix is of maximal rank and the Hessian criterion [21, Theorem 4.5] is satisfied must be singular points of the variety by [21, Lemma 4.4]. Characterizing the singular locus of secant varieties is a difficult problem. The approach we suggested based on the Hilbert function has the advantage that it sidesteps the problem of smoothness by proving that there are no isolated unidentifiable points when the assumptions of Proposition 38 are satisfied. It is an open question insofar the analysis of the Hilbert function may be more generally applicable for proving that the unidentifiable points must be contained in a curve; some results in this direction were established in $[8,9]$. Isolated unidentifiable tensors also exist in some cases, as was shown in [9, Example 3.4].

Acknowledgements. We thank I. Domanov, J. Migliore, and Y. Qi for helpful discussions. M. Mella is thanked for remarking that $S^{5} \mathbb{F}^{3}$ can be handled by the criterion in [45]. We thank two anonymous reviewers for their suggestions that improved the manuscript.

[1] H. Abo, G. Ottaviani, and C. Peterson, Induction for secant varieties of Segre varieties, Trans. Amer. Math. Soc., 361 (2009), pp. 767-792, doi:10.1090/S0002-9947-08-04725-9.

[2] J. Alexander and A. Hirschowitz, Polynomial interpolation in several variables, J. Algebraic Geom., 4 (1995), pp. 201-222.

[3] A. Anandkumar, R. Ge, D. Hsu, S. M. Kakade, and M. Telgarsky, Tensor decompositions for learning latent variable models, J. Mach. Learn. Res., 15 (2014), pp. 2773-2832.

[4] E. Angelini, C. Bocci, and L. Chiantini, Real identifiability vs complex identifiability, arXiv:1608.07197, (2016).

[5] C. Appellof AND E. DAvidSon, Strategies for analyzing data from video fluorometric monitoring of liquid chromatographic effluents, Anal. Chem., 53 (1981), pp. 2053-2056, 
doi:10.1021/ac00236a025.

[6] E. BALlico, On the weak non-defectivity of Veronese embeddings of projective spaces, Central Eur. J. Math., 3 (2005), pp. 183-187, doi:10.2478/BF02479194.

[7] E. Ballico AND A. Bernard, Decomposition of homogeneous polynomials with low rank, Mathematische Zeitshrift, 271 (2012), pp. 1141-1149, doi:10.1007/s00209-011-0907-6.

[8] E. Ballico AND L. ChiAntini, A criterion for detecting the identifiability of symmetric tensors of size three, Differential Geometry and Applic., 30 (2012), pp. 233-237, doi:10.1016/j.difgeo.2012.04.004.

[9] E. Ballico and L. Chiantini, Sets computing the symmetric tensor rank, Mediterr. J. Math., 10 (2013), pp. 643-654, doi:10.1007/s00009-012-0214-4.

[10] A. Bernardi, J. Brachat, P. Comon, And B. Mourrain, General tensor decomposition, moment matrices and applications, J. Symbolic Comput., 52 (2013), pp. 51-71, doi:10.1016/j.jsc.2012.05.012.

[11] A. Bhaskara, M. Charikar, A. Moitra, And A. Vijayaraghavan, Smoothed analysis of tensor decompositions, in STOC '14 Proceedings of the 46th Annual ACM Symposium on Theory of Computing, New York, NY, USA, 2014, pp. 594-603.

[12] A. Bigatti, A. V. Geramita, and J. C. Migliore, Geometric consequences of extremal behavior in a theorem of Macaulay, Trans. Amer. Math. Soc., 346 (1994), pp. 203-235, doi:10.1090/S0002-9947-1994-1272673-7.

[13] G. Blekherman And Z. Teitler, On maximum, typical, and generic ranks, Math. Ann., 362 (2015), pp. 1021-1031, doi:10.1007/s00208-014-1150-3.

[14] C. Bocci And L. Chiantini, On the identifiability of binary Segre products, J. Algebraic Geometry, 22 (2013), pp. 1-11, doi:10.1090/S1056-3911-2011-00592-4.

[15] C. Bocci, L. Chiantini, and G. Ottaviani, Refined methods for the identifiability of tensors, Ann. Mat. Pur. Appl. (4), 193 (2014), pp. 1691-1702, doi:10.1007/s10231-013-0352-8.

[16] J. Bochnak, M. Coste, And M. Roy, Real Algebraic Geometry, Springer-Verlag, 1998.

[17] J. Buczyński, A. Ginenski, And J. LAndsberg, Determinantal equations for secant varieties and the Eisenbud-Koh-Stillman conjecture, J. London Math. Soc., 88 (2013), pp. 1-24, doi:10.1112/jlms/jds073.

[18] M. Catalisano, A. Geramita, and A. Gimigliano, Ranks of tensors, secant varieties of Segre varieties and fat points, Linear Algebra Appl., 355 (2002), pp. 263-285, doi:10.1016/S00243795(02)00352-X.

[19] L. Chiantini And J. C. Migliore, Almost maximal growth of the Hilbert function, J. Algebra, 431 (2015), pp. 38-77, doi:10.1016/j.jalgebra.2015.01.030.

[20] L. Chiantini And G. Ottaviani, On generic identifiability of 3-tensors of small rank, SIAM J. Matrix Anal. Appl., 33 (2012), pp. 1018-1037, doi:10.1137/110829180.

[21] L. Chiantini, G. Ottaviani, and N. Vannieuwenhoven, An algorithm for generic and lowrank specific identifiability of complex tensors, SIAM J. Matrix Anal. Appl., 35 (2014), pp. 1265-1287, doi:10.1137/140961389.

[22] L. Chiantini, G. Ottaviani, and N. Vannieuwenhoven, On generic identifiability of symmetric tensors of subgeneric rank, Trans. Amer. Math. Soc., (2015).

[23] C. Ciliberto, Geometric Aspects of Polynomial Interpolation in More Variables and of Waring's Problem, vol. European Congress of Mathematics, Barcelona, July 10-14, 2000, Volume I of Progress in Mathematics, Birkhäuser Basel, 2001, doi:10.1007/978-3-0348-82682 217.

[24] P. Comon, G. H. Golub, L.-H. Lim, And B. Mourrain, Symmetric tensors and symmetric tensor rank, SIAM J. Matrix Anal. Appl., 30 (2008), pp. 1254-1279, doi:10.1137/060661569.

[25] L. De LATHAuWer, A link between the canonical decomposition in multilinear algebra and simultaneous matrix diagonalization, SIAM J. Matrix Anal. Appl., 28 (2006), pp. 642-666, doi:10.1137/040608830.

[26] I. Domanov And L. De Lathauwer, On the uniqueness of the canonical polyadic decomposition of third-order tensors-part II: Uniqueness of the overall decomposition, SIAM J. Matrix Anal. Appl., 34 (2013), pp. 876-903, doi:10.1137/120877258.

[27] I. Domanov And L. De Lathauwer, Canonical polyadic decomposition of third-order tensors: reduction to generalized eigenvalue decomposition, SIAM J. Matrix Anal. Appl., 35 (2014), pp. 636-660, doi:10.1137/130916084.

[28] I. Domanov and L. De Lathauwer, Generic uniqueness conditions for the canonical polyadic decomposition and INDSCAL, SIAM J. Matrix Anal. Appl., 36 (2015), pp. 1567-1589, doi: $10.1137 / 120877258$

[29] F. GalupPi AND M. Mella, Identifiability of homogeneous polynomials and Cremona transformations, arXiv:1606.06895, (2016).

[30] A. Geramita, M. Kreuzer, and L. Robbiano, Cayley-Bacharach schemes and their canonical 
modules, Trans. Amer. Math. Soc., 399 (1993), pp. 163-189, doi:10.1090/S0002-9947-19931102886-5.

[31] A. V. Geramita, T. Harima, and Y. Shin, An alternative to the Hilbert function for the ideal of a finite set of points in $\mathbb{P}^{n}$, Illinois J. Math., 45 (2001), pp. 1-23.

[32] D. Grayson and M. Stillman, Macaulay 2, a software system for research in algebraic geometry. www.math.uiuc.edu/Macaulay2. Last accessed July 6, 2016.

[33] J. D. Hauenstein, L. Oeding, G. Ottaviani, and A. J. Sommese, Homotopy techniques for tensor decomposition and perfect identifiability, arXiv:1501.00090, (2015).

[34] J. D. Hauenstein and J. I. Rodriguez, Numerical irreducible decomposition of multiprojective varieties, arXiv:1507.07069v1, (2015).

[35] A. Iarrobino and V. Kanev, Power Sums, Gorenstein Algebras, and Determinantal Loci, vol. 1721 of Lecture Notes in Mathematics, Springer, 1999.

[36] T. Jiang And N. D. Sidiropoulos, Kruskal's permutation lemma and the identification of CANDECOMP/PARAFAC and bilinear models with constant modulus constraints, IEEE Trans. Signal Process., 52 (2004), pp. 2625-2636, doi:10.1109/TSP.2004.832022.

[37] T. Jiang, N. D. Sidiropoulos, and J. M. F. Ten Berge, Almost-sure identifiability of multidimensional harmonic retrieval, IEEE Trans. Signal Process., 49 (2001), pp. 1849-1859, doi:10.1109/78.942615.

[38] J. B. KRUSKaL, Three-way arrays: rank and uniqueness of trilinear decompositions, with application to arithmetic complexity and statistics, Linear Algebra Appl., 18 (1977), pp. 95-138.

[39] J. Landsberg, The border rank of the multiplication of $2 \times 2$ matrices is seven, J. Amer. Math. Soc., 19 (2006), pp. 447-459, doi:10.1090/S0894-0347-05-00506-0.

[40] J. M. Landsberg, Tensors: Geometry and Applications, vol. 128 of Graduate Studies in Mathematics, AMS, Providence, Rhode Island, 2012.

[41] S. E. Leurgans, R. T. Ross, and R. B. Abel, A decomposition for three-way arrays, SIAM J. Matrix Anal. Appl., 14 (1993), pp. 1064-1083, doi:10.1137/0614071.

[42] M. Mella, Singularities of linear systems and the Waring problem, Trans. Amer. Math. Soc., 358 (2006), pp. 5523-5538, doi:10.1090/S0002-9947-06-03893-1.

[43] J. C. Migliore, Syzygies and Hilbert functions, in Lecture Notes in Pure and Applied Mathematics, I. Peeva, ed., vol. 254, Chapman \& Hall, Boca Raton, Florida, USA, 2007, ch. The geometry of Hilbert functions.

[44] J. Nie And L. WANG, Semidefinite relaxations for best rank-1 tensor approximations, SIAM J. Matrix Anal. Appl., 35 (2014), pp. 1155-1179, doi:10.1137/130935112.

[45] L. Oeding and G. Ottaviani, Eigenvectors of tensors and algorithms for Waring decomposition, J. Symbolic Comput., 54 (2013), pp. 9-35, doi:10.1016/j.jsc.2012.11.005.

[46] A. Phan, P. Tichavský, And A. Cichocki, CANDECOMP/PARAFAC decomposition of high-order tensors through tensor reshaping, IEEE Trans. Signal Process., (2013), doi:10.1109/TSP.2013.2269046.

[47] Y. QI, P. Comon, AND L.-H. Lim, Semialgebraic geometry of nonnegative tensor rank, arXiv:1601.05351, (2016).

[48] B. Reznick, Sums of even powers of real linear forms, Mem. Amer. Math. Soc., 96 (1992).

[49] J. Salmi, A. Richter, And V. Koivunen, Sequential unfolding SVD for tensors with applications in array signal processing, IEEE Trans. Signal Process., 57 (2009), pp. 4719-4733, doi:10.1109/TSP.2009.2027740.

[50] N. D. Sidiropoulos AND R. BRo, On the uniqueness of multilinear decomposition of n-way arrays, J. Chemometrics, 14 (2000), pp. 229-239.

[51] M. Sørensen and L. De Lathauwer, Coupled canonical polyadic decomposition and (coupled) decompositions in multilinear rank- $\left(l_{r, n}, l_{r, n}, 1\right)$ tterm-Part I: uniqueness, SIAM J. Matrix Anal. Appl., (2015), doi:10.1137/140956853.

[52] R. P. Stanley, Hilbert functions of graded algebras, Adv. in Math., 28 (1978), pp. 57-83.

[53] A. Stegeman, On uniqueness conditions for Candecomp/Parafac and Indscal with full column rank in one mode, Linear Algebra Appl., 431 (2009), pp. 211-227, doi:10.1016/j.laa.2009.02.025.

[54] V. Strassen, Rank and optimal computation of generic tensors, Linear Algebra Appl., 52-53 (1983), pp. 645-685, doi:10.1016/0024-3795(83)80041-X.

[55] A. Terracini, Sulla $V_{k}$ per cui la varietà degli $S_{h} h+1$-secanti ha dimensione minore dell'ordinario, Rend. Circ. Mat. Palermo, 31 (1911), pp. 392-396.

[56] N. Vannieuwenhoven, R. Vandebril, and K. Meerbergen, A new truncation strategy for the higher-order singular value decomposition, SIAM J. Sci. Comput., 34 (2012), pp. A1027A1052, doi:10.1137/110836067.

[57] T. Zhang AND G. H. Golub, Rank-one approximation to high order tensors, SIAM J. Matrix Anal. Appl., 23 (2001), pp. 534-550, doi:10.1137/S0895479899352045. 